Sharif University of Technology
Scientia Iranica
SCIENTIA
TRANactions A: Civil Engineering
IRItp://scientiairanica.sharif.edu

\title{
Predicting the effective stress parameter of unsaturated soils using adaptive neuro-fuzzy inference system
}

\author{
H. Rahnema ${ }^{a, *}$, M. Hashemi Jokar ${ }^{\mathrm{a}, 1}$, and H. Khabbaz ${ }^{\mathrm{b}}$ \\ a. Department of Civil and Environmental Engineering, Shiraz University of Technology, Shiraz, Iran. \\ b. School of Civil and Environmental Engineering, University of Technology, Sydney (UTS). \\ Received 7 March 2017; received in revised form 29 August 2017; accepted 19 February 2018
}

\author{
KEYWORDS \\ Unsaturated soils; \\ Effective stress \\ parameter; \\ ANFIA; \\ Fuzzy clustering; \\ Subtractive clustering; \\ FCM clustering.
}

\begin{abstract}
The effective stress parameter $(\chi)$ is applied to obtain the shear strength of unsaturated soils. In this study, two Adaptive Neuro-Fuzzy Inference System (ANFIS) models, including SC-FIS model (created by subtractive clustering) and FCM-FIS model (created by Fuzzy c-means (FCM) clustering), are presented for prediction of $\chi$, and the results are compared. The soil-water characteristic curve fitting parameter $(\lambda)$, the confining pressure, the suction, and the volumetric water content in dimensionless forms are used as input parameters for these two models. By using a trial-and-error process, a series of analyses were performed to determine the optimum methods. The ANFIS models were constructed, trained, and validated to predict the value of $\chi$. The quality of the ANFIS prediction ability was quantified in terms of the determination coefficient $\left(R^{2}\right)$, Root Mean Square Error (RMSE), and Mean Absolute Error (MAE). These two ANFIS models are able to effectively predict the value of $\chi$ with reasonable values of $R^{2}$, RMSE, and MAE. Sensitivity analysis was implemented to determine the effect of input parameters on $\chi$ prediction, and the results revealed that the confining pressure and the volumetric water content parameters had the most influence on $\chi$ prediction.
\end{abstract}

(C) 2019 Sharif University of Technology. All rights reserved.

\section{Introduction}

Compacted soils, which are commonly used in geotechnical engineering projects, such as earth dams, highways, embankments, and airport runways, are mostly unsaturated. To achieve a safe design in all these projects, the stress state variable in soil plays a significant role. Any proposed model for the stress state variable should express its independence from the soil

1. Present address: Graduate University of Advanced Technology, Kerman, Iran.

*. Corresponding author. Tel/Fax: +987137277656 E-mail addresses: rahnema@sutech.ac.ir (H. Rahnema); m.hashemijokar@sutech.ac.ir (M. Hashemi Jokar); Hadi.Khabbaz@uts.edu.au (H.Khabbaz)

doi: $10.24200 /$ sci. 2018.20200 properties [1]. In saturated soils, the effective stress is taken into account as the stress state variable [2]. Some researchers have attempted to find the stress state variable for unsaturated soils the same as that for saturated soils with only one variable; however, they have noticed that the soil properties have been involved in the proposed models [3-6]. Therefore, in unsaturated soil, the stress state variable consists of two stress state variables [4]:

$$
\sigma^{\prime}=\left(\sigma-u_{a}\right)+\chi\left(u_{a}-u_{w}\right),
$$

where $\chi$ is the effective stress parameter. Parameter $\chi$ varies from 1 to 0 from saturated to dry soils, respectively, $u_{a}$ is the pore air pressure, $\left(\sigma-u_{a}\right)$ is the net normal stress, and $\left(u_{a}-u_{w}\right)$ is the matric suction denoted by $S$. Khalili and Khabbaz [7] solved $\chi$ as a function of suction ratio as follows: 


$$
\chi= \begin{cases}\left(\frac{u_{a}-u_{w}}{u_{e}}\right)^{-0.55} & \text { for } u_{a}-u_{w}>u_{e} \\ 1 & \text { for } u_{a}-u_{w} \leq u_{e}\end{cases}
$$

where $u_{e}$ (the air entry value) is a measure of suction, showing the transition from the saturated state to the unsaturated state. Determining all of these parameters to quantify the value of $\chi$ is a difficult and timeconsuming task and needs conducting many laboratory tests. In addition, theoretical studies have also shown that $\chi$ is highly nonlinear and may exceed unity [8].

The shear strength of unsaturated soil may be determined by means of the concept of the stress state variable. Bishop [4] proposed the following equation to calculate the shear strength of unsaturated soils:

$$
\tau=c^{\prime}+\left(\sigma-u_{a}\right) \tan \phi^{\prime}+\chi S \tan \phi^{\prime},
$$

where $c^{\prime}$ and $\phi^{\prime}$ are the effective cohesion and the internal friction angle, respectively.

According to the shear strength of saturated soils, Eq. (3) is presented by Fredlund et al. [9] to calculate the shear strength of unsaturated soils.

$$
\tau=c^{\prime}+\left(\sigma-u_{a}\right) \tan \phi^{\prime}+S \tan \phi^{b},
$$

where $\phi^{b}$ is the friction angle associated with changes in $S$ alone. The relationship between $\phi^{\prime}$ and $\phi^{b}$ is presented by Escario and Saez [10] as follows:

$$
\chi=\frac{\tan \phi^{b}}{\tan \phi^{\prime}} .
$$

$\chi$ is generally assumed to be a function of the degree of saturation $\left(S_{r}\right)$. This parameter has been proposed through the best-fit regression formulas conducted on some suitable experimental data. In the following equations, $\chi$ is presented as a function of $S_{r}$, which is obtained from the best fit of the experimental results $[11,12]$ :

$$
\begin{aligned}
& \chi=S_{r}^{k}=\left(\frac{\theta}{\theta_{s}}\right)^{k} \\
& \chi=\frac{S_{r}-S_{r r}}{1-S_{r r}}=\frac{\theta-\theta_{r}}{\theta_{s}-\theta_{r}},
\end{aligned}
$$

where $S_{r r}$ is the residual degree of saturation (the saturation of water around the soil particle surfaces exists like tine films), $S_{r}$ is the degree of saturation (at the moment of testing), $\theta, \theta_{s}$, and $\theta_{r}$ are the volumetric, saturated, and residual volumetric water contents, respectively, and $k$ is an optimized parameter, determined from the best fit between measured and predicted values. Figure 1 shows $S_{r}$ versus $\chi$ for the various values of $k$ and $S_{r r}$ [8].

An accurate prediction of $\chi$ can be achieved when improved approaches are utilized for a nonlinear

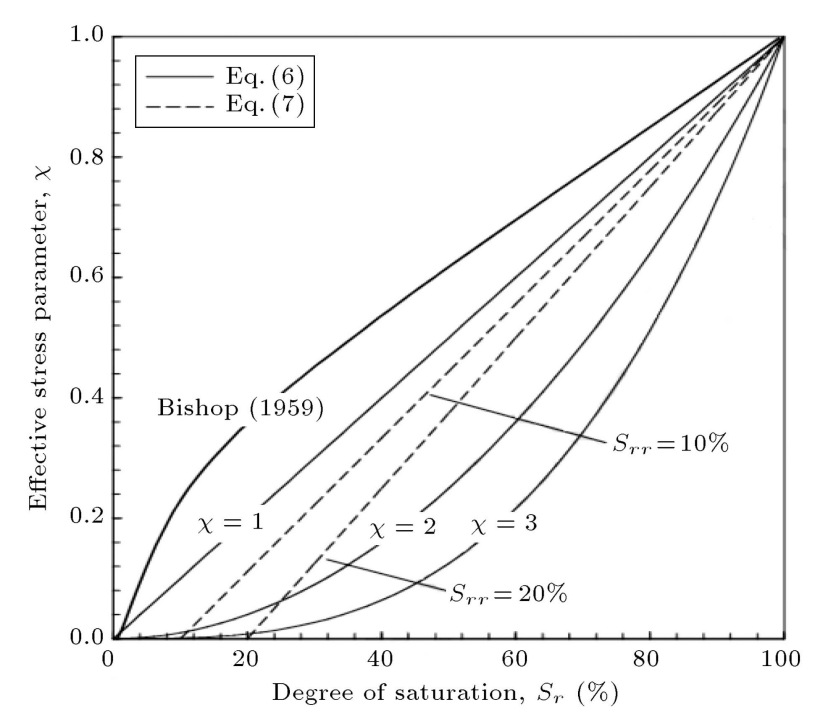

Figure 1. $S_{r}$ versus $\chi$ for various values of $k$ and $S_{r r}$ [8].

problem. Artificial Intelligence (AI) based techniques have been useful as an alternative approach to replace the conventional techniques for the prediction of engineering problems. Some AI-based models have been recently used for the prediction of $\chi$ based on available empirical data [13-15].

Due to the nonlinearity of the relationship between $\chi$ and related parameters, the prediction process can be complex. Therefore, a powerful model, such as the Adaptive Neuro-Fuzzy Inference System (ANFIS) model, should be employed to predict the process accurately. ANFIS models are well-known hybrid neurofuzzy networks for modeling complex systems [16]. Characterized by the learning capability from past experiences, ANFIS is going to be one of the pillars of scientific research [17].

In the field of geotechnical engineering, the use of ANFIS is also in progress. Gokceoglu et al. [18] used the neuro-fuzzy system to estimate the deformation modulus of rock masses, and their neurofuzzy model exhibited a high performance. Kalkan et al. [19] developed ANFIS and Artificial Neural Network (ANN) models to predict the Unconfined Compressive Strength (UCS) of compacted granular soils, and the results of the ANFIS model were very encouraging, compared to the ANN model. Kayadelen et al. [20] used ANFIS to predict the swell percentage of compacted soils, and showed that ANFIS was a more reasonable method for predicting the swelling potential of soils. Cobaner [21] predicted the estimation of evapotranspiration (ET0) using climatic variables by two ANFIS models, based on grid partition (G-ANFIS) and subtractive clustering (S-ANFIS), and Multi-Layer Perceptron (MLP) model; it was found that S-ANFIS model showed better results than G-ANFIS and MLP models. Sezer et al. [22] successfully trained an ANFIS model to predict permeability based on 20 different 
types of granular soils. Ikizler et al. [23] showed that neural network and adaptive neuro-fuzzy-based prediction models could satisfactorily be used to obtain the swelling pressure of expansive soils. Doostmohammadi [24] used ANFIS model to predict time-dependent swelling pressure (SPf), compared the ANFIS results with ANN and multiple regression approaches, and proved that the ANFIS model was more effective in modeling the cyclic swelling pressure. Cabalar et al. [25] developed ANFIS models for (I) damping ratio and shear modulus of coarse rotund sand-mica mixtures based on experimental results from Stokoe's resonant column testing apparatus, (II) deviatoric stressstrain, pore water pressure generation-strain properties of coarse rotund sand-mica mixtures from triaxial testing apparatus, and (III) liquefaction triggering. Zoveidavianpoor [26] compared the capability levels of ANN and ANFIS for the prediction of compressional wave ( $p$ wave) velocity, and proved that ANFIS and ANN systems performed comparably well and accurate for the prediction of $\mathrm{p}$ wave.

\section{Adaptive Neuro-Fuzzy Inference System (ANFIS)}

Fuzzy Logic (FL), introduced by Zadeh [27], is commonly applied with investigative knowledge and imprecise inputs to realize complicated functions. The membership grade with the numbers 0 or 1 is expressed in the classical logic; however, FL can have any number between 0 and 1. Membership grade of each member is determined by the membership functions. Fuzzy Inference System (FIS) is the same as a black box, which connects the input space to the output space through some fuzzy if-then rules. A fuzzy if-then rule can be shown as follows:

if $x$ is $A$ then $y$ is $B$,

where $A$ and $B$ are linguistic values defined by fuzzy sets on the ranges (universes of discourse) $X$ and $Y$, respectively. " $x$ is $A$ " is called antecedent or premise, and " $y$ is $B$ " is called consequent or conclusion [28]. Three well-known FIS models include Mamdani and Assilian [29], Takagi-Sugeno-Kang (TSK) [30], and Tsukamoto [31].

Neural Networks (NNs) [32] can learn from historical data and train themselves to achieve high performance, while extensive expertise is not mandatory. Among the most powerful data-driven methods, FL and NNs systems are able to monitor data pattern classification in diagnostic tasks. Adaptive NeuroFuzzy Inference System (ANFIS) [16] is a combination of FL and NNs. ANFIS applied a Takagi-Sugeno-Kang Fuzzy Inference System (TSK-FIS) to a set of input and output data. TSK-FIS if-then rules are simply presented as follows [33]:

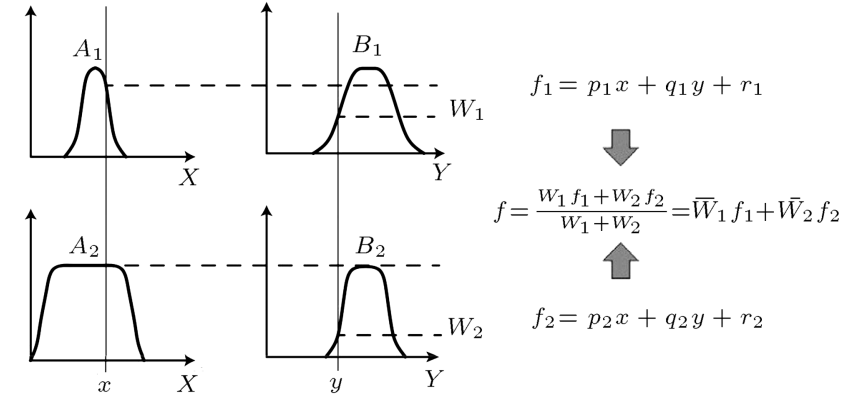

Figure 2. A typical fuzzy model of TS type [16].

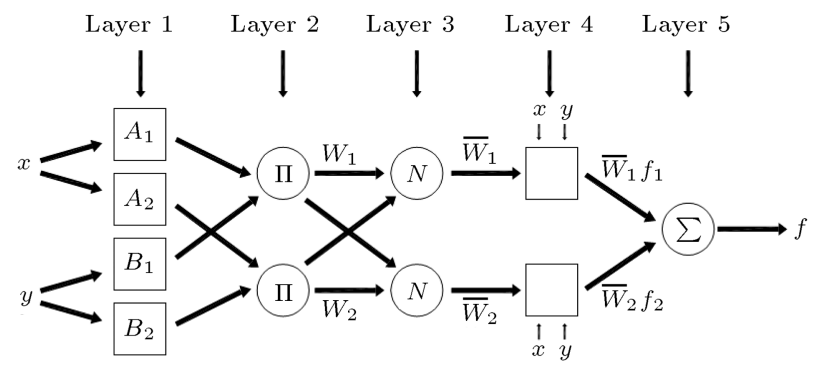

Figure 3. A typical ANFIS structure [58].

Rule 1 : if $x$ is $A_{1}, y$ is $B_{1}$ then $f_{1}=p_{1} x+q_{1} y+r_{1}$,

Rule $2:$ if $x$ is $A_{2}, y$ is $B_{2}$ then $f_{2}=p_{2} x+q_{2} y+r_{2}$,

where $A_{i}$ and $B_{i}$ are the linguistic labels of the $i$ th rule. $p_{i}, q_{i}$, and $r_{i}$ are the consequent parameters in the TSK-FIS [28].

TSK-FIS with 2 inputs ( $x$ and $y$ ) and one output $(f)$ is presented in Figure 2.

ANFIS, as an optimization method, uses hybrid learning algorithms (gradient descent and least-squares method). The antecedent and consequent parameters will be adjusted with gradient descent and least-squares method, respectively [33]. Figure 3 shows the ANFIS structure that has five layers of nodes including square nodes (adaptive nodes whose parameters will vary during training) and circle nodes (fixed nodes whose parameters will not vary during training).

The layers of ANFIS are described as follows [16]:

- In Layer 1, the fuzzy membership grade of the inputs may be obtained through Eq. (8) as follows:

$$
\begin{aligned}
& O_{1, i}=\mu_{A i}(x) \quad i=1,2, \\
& O_{1, i}=\mu_{B(i-2)}(x) \quad i=3,4,
\end{aligned}
$$

where $\mu_{A i}$ and $\mu_{B i}$ are the grades of membership functions of $A_{i}$ and $B_{i}$, respectively, and are defined by the membership function;

- In Layer 2, the output of each node is multiplied by the input signals and represents the firing strength (the degree that the antecedent part of a fuzzy rule 
is fulfilled) of a law.

$$
O_{2, i}=w_{i}=\mu_{A i}(x) \mu_{B i}(y) \quad i=1,2,
$$

where $w_{i}$ is the firing strength of the $i$ th rule.

- In Layer 3 , the normalized firing strength, $\bar{w}_{i}$, is presented as follows:

$$
O_{3, i}=\bar{w}_{i}=\frac{w_{i}}{w_{1}+w_{2}} .
$$

- In Layer 4, the contribution of the $i$ th rule in the output is calculated with an adaptive function:

$$
O_{4, i}=\bar{w}_{i} f_{i}=\bar{w}_{i}\left(p_{i} x+q_{i} y+r_{i}\right),
$$

where $f_{i}$ is the linear function of the inputs.

- Finally, in Layer 5, the summation of all input signals is calculated as follows:

$$
O_{5, i}=\sum_{i} \bar{w}_{i} f_{i}=\frac{\sum_{i} w_{i} f_{i}}{\sum_{i} w_{i}}
$$

In this paper, to determine the membership grade, the Gaussian membership function is used as follows:

$$
\mu_{A i}\left(x_{j}\right)=e^{-\left(\frac{x_{j}-c_{i}^{j}}{\sigma_{i}^{j}}\right)^{2}}
$$

where $x_{j}$ is the input data variable; $c_{i}^{j}$ and $\sigma_{i}^{j}$ are the center (mean) and the width (standard deviation) of the membership function, respectively. Actually, $c_{i}^{j}$ and $\sigma_{i}^{j}$ are the antecedent parameters of fuzzy rules for the Gaussian membership function.

\section{Fuzzy clustering}

Fuzzy clustering of data provides a division of the data space into fuzzy clusters and gives useful information by grouping data from a large dataset that represents a system behavior. In this way, each obtained cluster center represents a rule. There are several methods for clustering such as $k$-means clustering [34], fuzzy c-means clustering [35], and mountain and subtractive clustering method, which is a non-iterative algorithm [36]. In this paper, the initial FIS for ANFIS models is used, which is created by subtractive and fuzzy c-means clustering methods.

\subsection{Subtractive clustering}

In 1994, the Subtractive Clustering (SC) model was developed by Chiu [36,37]. This model is a fast, robust, and accurate algorithm for specifying the number of clusters and the cluster centers in a set of data. SC is an extension of the grid-based mountain clustering method [38]. Based on the density of surrounding data points, in the SC method, each data point is assumed to be a likely cluster center, and its potential is then calculated. The steps of the SC process for a collection of $n$ data points, $Y=\left\{y_{1}, y_{2}, \ldots, y_{n}\right\}$, in $d$-dimensional space can be summarized as follows [36]:
1. Normalize the dataset between 0 and 1 by calculating the following formulation for each dimension of data:

$$
x_{i}^{l}=\frac{y_{i}^{l}-y_{\min }^{l}}{y_{\max }^{l}-y_{\min }^{l}} \quad i=1,2, \ldots, n \quad l=1,2, \ldots, d,
$$

where $x_{i}^{l}$ is the normalized value of the $i$ th data in the $l$ th dimension, $y_{i}^{l}$ is the $i$ th data in the $l$ th dimension, and $y_{\min }^{l}$ and $y_{\max }^{l}$ are the minimum and maximum values of data samples in the $l$ th dimension.

2. Determine a potential value at each data point, $x_{i}$ :

$$
\begin{aligned}
& p_{i}=\sum_{j=1}^{n} e^{-\alpha\left\|x_{i}-x_{j}\right\|^{2}}, \\
& \alpha=\frac{4}{r_{a}^{2}}
\end{aligned}
$$

where $\|$.$\| is the Euclidean distance, x_{i}$ and $x_{j}$ are the normalized data points with $d$-dimensional space, and $r_{a}$ is the positive constant between 0 and 1 , representing a neighborhood radius.

The data points with many neighboring data points will have high potential value. The first cluster center is chosen as the data point with the maximum potential value among all other data points;

3. Calculate the reduction potential value of each remaining data point as follows:

$$
\begin{aligned}
& p_{i}=p_{i}-p_{1}^{*} e^{-\beta\left\|x_{i}-x_{1}^{*}\right\|^{2}}, \\
& \beta=\frac{4}{r_{b}^{2}} \\
& r_{b}=\eta r_{a},
\end{aligned}
$$

where $x_{1}^{*}$ is the first cluster center, $p_{1}^{*}$ is its potential value, and $\eta$ is the squash factor with a constant value greater than 1 .

The second cluster center is chosen as a data point with the maximum remaining potential;

4. Find the other cluster centers using the following equation:

$$
p_{i}=p_{i}-p_{k}^{*} e^{-\beta\left\|x_{i}-x_{k}^{*}\right\|^{2}},
$$

where $x_{c}^{*}$ is the $k$ th cluster center, and $p_{k}^{*}$ is its potential value data as a cluster center. Some conditions, such as Eqs. (21) to (23), must be checked in the SC process [33]:

$$
\begin{aligned}
& p_{k}^{*}>\bar{\varepsilon} p_{1}^{*}, \\
& p_{k}^{*}<\varepsilon p_{1}^{*},
\end{aligned}
$$




$$
\frac{d_{m i n}}{r_{a}}+\frac{p_{k}^{*}}{p_{1}^{*}} \geq 1
$$

where $\bar{\varepsilon}$ and $\underline{\varepsilon}$ are accepted and rejected ratios, respectively, and $d_{\min }$ is the shortest distance between $x_{k}^{*}$ and all the previously found cluster centers. $x_{k}^{*}$, as a cluster center, will be accepted, when Eq. (21) is satisfied; the clustering process will be completed if Eq. (22) is satisfied; consequently, Eq. (23) should be satisfied. Chiu [36] suggested that $\bar{\varepsilon}=0.5$ and $\underline{\varepsilon}=0.15$. The fourth step will be repeated until the above conditions are fulfilled and, then, the SC process is accomplished. The number of clusters and fuzzy rules is equal and will be changed by the value of $r_{a}$. The great value of $r_{a}$ makes a fewer number of cluster centers, and vice versa [33].

\subsection{Fuzzy c-means clustering}

Fuzzy c-means (FCM) clustering was developed by Dunn [39] and improved by Bezdek [35]. In the FCM clustering, firstly, the number of clusters is chosen, and the sample data points are clustered into the chosen cluster numbers. This method can obtain the cluster centers directly. Let $Y=\left\{y_{1}, y_{2}, \ldots, y_{n}\right\}$ be the sample data points, and each data point has $d$ dimensions. These data points should be clustered into $\mathrm{C}$ clusters. The objective function for FCM is defined as follows:

$$
J_{m}=\sum_{i=1}^{n} \sum_{j=1}^{c} u_{i j}^{m}\left\|y_{i}-c_{j}\right\|^{2},
$$

where $m$ is the weighting exponent and has a constant value greater than 1 . Bezdek [35] suggested that $m=2$, $y_{i}$ is the $i$ th data point, $c_{j}$ is the $j$ th center of cluster, $\|$.$\| is the Euclidean distance between y_{i}$ and $c_{j}$, and $u_{i j}$ is the membership degree of the $i$ th data point in the $j$ th cluster.

$u_{i j}$ and $c_{j}$ are calculated by the following equations [33]:

$$
\begin{aligned}
& u_{i j}=\frac{1}{\sum_{k=1}^{c}\left(\frac{\left\|y_{i}-c_{j}\right\|}{\left\|y_{i}-c_{k}\right\|}\right)^{\frac{2}{m-1}}}, \\
& c=\frac{\sum_{i=1}^{n} u_{i j}^{m} * y_{i}}{\sum_{i=1}^{n} u_{i j}^{m}}
\end{aligned}
$$

In the clustering process, Eqs. (25) and (26) will be updated until the stopping condition (Eq. (27)) is fulfilled.

$$
\max \left\{\left|u_{i j}^{(k+1)}-u_{i j}^{(k)}\right|\right\}<\varepsilon
$$

where $\varepsilon$ is a criterion value to stop clustering, and $k$ is the iteration step.

The above formulation allows the objective function $\left(J_{m}\right)$ to converge to the possible minimum value [33].

\section{Used database for modeling}

In this study, the datasets used to develop two ANFIS models were derived from 120 collected data from the literature [40-49]. These data were associated with the results of triaxial, shear, pressure plate, and filter paper tests. These datasets consist of seven characteristics of unsaturated soils: suction $(S)$, bubbling pressure $\left(h_{b}\right)$, net confining pressure $(P)$, residual water content $\left(\theta_{r}\right)$, saturated volumetric water content $\left(\theta_{s}\right)$, soilwater characteristic curve fitting parameter $(\lambda)$, and effective stress parameter $(\chi) . S, h_{b}, P, \theta_{r}$, and $\theta_{s}$ characteristics of data became dimensionless as follows: $\frac{P}{P_{0}}$ is the dimensionless confining pressure parameter $P_{0}=101.325 \mathrm{kPa}, \frac{S}{h_{b}}$ is the dimensionless suction parameter, and $\frac{\theta_{r}}{\theta_{s}}$ is the dimensionless volumetric water content parameter (Table 1). Table 2 shows the range of $\frac{P}{P_{0}}, \frac{S}{h_{b}}, \frac{\theta_{r}}{\theta_{s}}$, and $\lambda$.

The datasets were divided into three separate groups: the training dataset (used to train the ANFIS model) by 85 data (71\%), the validation dataset (used to prevent overfitting through training procedure) by 15 data (12\%), and the testing dataset (used to verify the accuracy and effectiveness of the model) by 20 data $(17 \%)$. The data were chosen randomly in each dataset.

\section{Developing ANFIS models to predict $\chi$ value}

In order to develop the ANFIS model, firstly, the initial Fuzzy Inference System (FIS) was created and, then, trained by ANFIS. In this paper, two initial FIS models, SC-FIS and FCM-FIS, were created by the application of Subtractive Clustering (SC) and Fuzzy cmeans (FCM) clustering, respectively. In other words, the TSK-FIS in these models was created by the SC and FCM clustering, respectively. The fuzzy if-then rules in TSK-FIS models for predicting $\chi$ are defined as follows:

$$
\text { Rulec }:\left\{\begin{array}{c}
\text { If } x_{1} \text { is } A_{1 c} \text { and } x_{2} \text { is } A_{2 c} \text { and } x_{3} \text { is } A_{3 c} \\
\text { and } x_{4} \text { is } A_{4 c} \\
\text { Then } f_{c}=p_{0 c}+p_{1 c} x_{1}+p_{2 c} x_{2} \\
\quad+p_{3 c} x_{3}+p_{4 c} x_{4}
\end{array}\right.
$$

where $x_{1}, x_{2}, x_{3}$, and $x_{4}$ are $\frac{S}{h_{b}}, \frac{P}{P_{0}}, \frac{\theta_{r}}{\theta_{s}}$, and $\lambda$, respectively. $c$ is the cluster number $(c=1 \sim C)$, $A_{1 c}, A_{2 c}, A_{3 c}$, and $A_{4 c}$ are the linguistic labels (the fuzzy membership functions) of the $c$ th rule, $f_{c}$ is the consequent of Rule $c$, and $p_{0 c}, p_{1 c}, p_{2 c}, p_{3 c}$, and $p_{4 c}$ are the consequent parameters for the $c$ th rule. As mentioned earlier, the number of the clusters is equal to that of fuzzy rules and membership functions. To achieve the best solution to the problem with high 
Table 1. The dimensionless data used for modeling $\chi$.

\begin{tabular}{|c|c|c|c|c|c|c|}
\hline Data no. & Reference & $\frac{P}{P_{0}}$ & $\frac{S}{h_{b}}$ & $\frac{\theta_{r}}{\theta_{s}}$ & $\lambda$ & $\chi$ \\
\hline 1 & {$[40]$} & 0.493462 & 0.5 & 0.005166 & 0.89 & 1 \\
\hline 2 & {$[40]$} & 0.986923 & 0.5 & 0.005166 & 0.89 & 1 \\
\hline 3 & [40] & 1.480385 & 0.5 & 0.005166 & 0.89 & 1 \\
\hline 4 & {$[40]$} & 0.493462 & 1 & 0.005166 & 0.89 & 1 \\
\hline 5 & {$[40]$} & 0.986923 & 1 & 0.005166 & 0.89 & 1 \\
\hline 6 & {$[40]$} & 1.480385 & 1 & 0.005166 & 0.89 & 1 \\
\hline 7 & {$[40]$} & 0.493462 & 1.5 & 0.005166 & 0.89 & 0.7024 \\
\hline 8 & {$[40]$} & 0.986923 & 1.5 & 0.005166 & 0.89 & 0.6737 \\
\hline 9 & {$[40]$} & 1.480385 & 1.5 & 0.005166 & 0.89 & 0.8582 \\
\hline 10 & {$[41]$} & 0 & 0 & 0.224028 & 0.62 & 1 \\
\hline 11 & {$[41]$} & 1.480385 & 0 & 0.224028 & 0.62 & 1 \\
\hline 12 & {$[41]$} & 2.96077 & 0 & 0.224028 & 0.62 & 1 \\
\hline 13 & {$[41]$} & 0 & 20 & 0.224028 & 0.62 & 0.3562 \\
\hline 14 & {$[41]$} & 1.480385 & 20 & 0.224028 & 0.62 & 0.3733 \\
\hline 15 & {$[41]$} & 2.96077 & 20 & 0.224028 & 0.62 & 0.3678 \\
\hline 16 & {$[41]$} & 0 & 40 & 0.224028 & 0.62 & 0.2348 \\
\hline 17 & {$[41]$} & 1.480385 & 40 & 0.224028 & 0.62 & 0.3228 \\
\hline 18 & {$[41]$} & 2.96077 & 40 & 0.224028 & 0.62 & 0.3541 \\
\hline 19 & {$[41]$} & 0 & 80 & 0.224028 & 0.62 & 0.1741 \\
\hline 20 & {$[41]$} & 1.480385 & 80 & 0.224028 & 0.62 & 0.2465 \\
\hline 21 & {$[41]$} & 2.96077 & 80 & 0.224028 & 0.62 & 0.2905 \\
\hline 22 & [41] & 0 & 120 & 0.224028 & 0.62 & 0.1463 \\
\hline 23 & {$[41]$} & 1.480385 & 120 & 0.224028 & 0.62 & 0.1832 \\
\hline 24 & {$[41]$} & 2.96077 & 120 & 0.224028 & 0.62 & 0.2504 \\
\hline 25 & {$[49]$} & 0 & 51 & 0 & 0.19 & 0.11 \\
\hline 26 & {$[49]$} & 0 & 12.86486 & 0 & 0.19 & 0.41 \\
\hline 27 & [49] & 0 & 7.810811 & 0 & 0.19 & 0.6 \\
\hline 28 & [49] & 0 & 4.135135 & 0 & 0.19 & 0.74 \\
\hline 29 & [49] & 0 & 24.35135 & 0 & 0.19 & 0.27 \\
\hline 30 & {$[49]$} & 0 & 2.205405 & 0 & 0.19 & 0.9 \\
\hline 31 & {$[49]$} & 0 & 2.756757 & 0 & 0.19 & 0.79 \\
\hline 32 & [49] & 2.013323 & 82.7027 & 0 & 0.19 & 0.24 \\
\hline 33 & [49] & 2.013323 & 71.21622 & 0 & 0.19 & 0.26 \\
\hline 34 & [49] & 2.013323 & 67.08108 & 0 & 0.19 & 0.35 \\
\hline 35 & {$[49]$} & 2.013323 & 61.56757 & 0 & 0.19 & 0.32 \\
\hline 36 & {$[49]$} & 2.013323 & 59.72973 & 0 & 0.19 & 0.49 \\
\hline 37 & {$[49]$} & 2.013323 & 28.94595 & 0 & 0.19 & 0.53 \\
\hline 38 & [49] & 2.013323 & 5.972973 & 0 & 0.19 & 0.9 \\
\hline 39 & [49] & 0 & 1.064877 & 0.619678 & 0.96 & 0.47 \\
\hline 40 & {$[49]$} & 0 & 0.608501 & 0.619678 & 0.96 & 1 \\
\hline 41 & [49] & 0 & 0.760626 & 0.619678 & 0.96 & 1 \\
\hline 42 & {$[49]$} & 0 & 0.380313 & 0.619678 & 0.96 & 1 \\
\hline 43 & [49] & 0 & 0.456376 & 0.619678 & 0.96 & 1 \\
\hline 44 & [49] & 0 & 0.281432 & 0.619678 & 0.96 & 1 \\
\hline 45 & {$[49]$} & 0 & 0.304251 & 0.619678 & 0.96 & 1 \\
\hline
\end{tabular}


Table 1. The dimensionless data used for modeling $\chi$ (continued).

\begin{tabular}{|c|c|c|c|c|c|c|}
\hline Data no. & Reference & $\frac{P}{P_{0}}$ & $\frac{S}{h_{b}}$ & $\frac{\theta_{r}}{\theta_{s}}$ & $\lambda$ & $\chi$ \\
\hline 46 & {$[49]$} & 0 & 0.684564 & 0.619678 & 0.96 & 1 \\
\hline 47 & [43] & 1.973847 & 1.052632 & 0.6845 & 0.48 & 0.6462 \\
\hline 48 & [43] & 1.973847 & 2.105263 & 0.6845 & 0.48 & 0.5755 \\
\hline 49 & [43] & 1.973847 & 0.8 & 0.713746 & 0.91 & 1 \\
\hline 50 & [43] & 1.973847 & 1.6 & 0.713746 & 0.91 & 0.6883 \\
\hline 51 & [43] & 1.973847 & 3.2 & 0.713746 & 0.91 & 0.57 \\
\hline 52 & [43] & 1.973847 & 0.5 & 0.633816 & 1.05 & 1 \\
\hline 53 & {$[43]$} & 1.973847 & 1 & 0.633816 & 1.05 & 1 \\
\hline 54 & {$[43]$} & 1.973847 & 2 & 0.633816 & 1.05 & 0.7283 \\
\hline 55 & {$[43]$} & 0.296077 & 8 & 0.137 & 1.11 & 0.8938 \\
\hline 56 & [43] & 0.296077 & 24 & 0.137 & 1.11 & 0.4966 \\
\hline 57 & {$[43]$} & 0.296077 & 40 & 0.137 & 1.11 & 0.3575 \\
\hline 58 & {$[43]$} & 1.233654 & 8 & 0.137 & 1.11 & 0.8938 \\
\hline 59 & [43] & 1.233654 & 24 & 0.137 & 1.11 & 0.6952 \\
\hline 60 & [43] & 1.233654 & 40 & 0.137 & 1.11 & 0.5363 \\
\hline 61 & {$[43]$} & 2.467308 & 8 & 0.137 & 1.11 & 1 \\
\hline 62 & [43] & 2.467308 & 24 & 0.137 & 1.11 & 0.9931 \\
\hline 63 & [43] & 2.467308 & 40 & 0.137 & 1.11 & 0.5959 \\
\hline 64 & [43] & 0.296077 & 2.857143 & 0 & 0.41 & 0.7151 \\
\hline 65 & [43] & 0.296077 & 8.571429 & 0 & 0.41 & 0.4966 \\
\hline 66 & [43] & 0.296077 & 14.28571 & 0 & 0.41 & 0.4052 \\
\hline 67 & [43] & 1.233654 & 8.571429 & 0 & 0.41 & 0.8541 \\
\hline 68 & {$[43]$} & 1.233654 & 2.857143 & 0 & 0.41 & 1 \\
\hline 69 & {$[43]$} & 1.233654 & 14.28571 & 0 & 0.41 & 0.7151 \\
\hline 70 & {$[43]$} & 2.467308 & 2.857143 & 0 & 0.41 & 0.8938 \\
\hline 71 & {$[43]$} & 2.467308 & 8.571429 & 0 & 0.41 & 0.8938 \\
\hline 72 & [43] & 2.467308 & 14.28571 & 0 & 0.41 & 0.7151 \\
\hline 73 & [44] & 0.197385 & 2.666667 & 0.505987 & 0.33 & 0.5795 \\
\hline 74 & [44] & 0.690846 & 2.666667 & 0.505987 & 0.33 & 0.7671 \\
\hline 75 & {$[44]$} & 1.184308 & 2.666667 & 0.505987 & 0.33 & 1 \\
\hline 76 & {$[44]$} & 0.296077 & 1.666667 & 0.505987 & 0.33 & 0.8717 \\
\hline 77 & [44] & 0.493462 & 1.666667 & 0.505987 & 0.33 & 1 \\
\hline 78 & {$[44]$} & 0.986923 & 1.666667 & 0.505987 & 0.33 & 1 \\
\hline 79 & [44] & 0.789539 & 4 & 0.505987 & 0.33 & 0.8494 \\
\hline 80 & {$[44]$} & 1.283 & 4 & 0.505987 & 0.33 & 0.7938 \\
\hline 81 & [44] & 0.197385 & 6.666667 & 0.505987 & 0.33 & 1 \\
\hline 82 & {$[44]$} & 0.493462 & 6.666667 & 0.505987 & 0.33 & 1 \\
\hline 83 & {$[44]$} & 0.986923 & 6.666667 & 0.505987 & 0.33 & 1 \\
\hline 84 & {$[44]$} & 0.296077 & 4 & 0.505987 & 0.33 & 0.604 \\
\hline 85 & {$[46]$} & 0.986923 & 200 & 0.655157 & 0.21 & 0.6185 \\
\hline 86 & [46] & 3.947693 & 200 & 0.655157 & 0.21 & 0.6098 \\
\hline 87 & {$[46]$} & 3.947693 & 200 & 0.655157 & 0.21 & 0.2525 \\
\hline 88 & {$[46]$} & 3.947693 & 300 & 0.655157 & 0.21 & 0.5308 \\
\hline 89 & {$[47,48]$} & 0.493462 & 8.333333 & 0.272727 & 8.33 & 0.731 \\
\hline 90 & {$[47,48]$} & 0.986923 & 8.333333 & 0.325 & 11.82 & 1 \\
\hline 91 & {$[47,48]$} & 0.493462 & 16.66667 & 0.272727 & 8.33 & 0.497 \\
\hline
\end{tabular}


Table 1. The dimensionless data used for modeling $\chi$ (continued).

\begin{tabular}{|c|c|c|c|c|c|c|}
\hline Data no. & Reference & $\frac{P}{P_{0}}$ & $\frac{S}{h_{b}}$ & $\frac{\theta_{r}}{\theta_{s}}$ & $\lambda$ & $\chi$ \\
\hline 92 & {$[47,48]$} & 0.986923 & 16.66667 & 0.325 & 11.82 & 0.694 \\
\hline 93 & {$[47,48]$} & 0.493462 & 33.33333 & 0.272727 & 8.33 & 0.239 \\
\hline 94 & {$[47,48]$} & 0.986923 & 33.33333 & 0.325 & 11.82 & 0.337 \\
\hline 95 & {$[47,48]$} & 0.493462 & 66.66667 & 0.272727 & 8.33 & 0.091 \\
\hline 96 & {$[47,48]$} & 0.986923 & 66.66667 & 0.325 & 11.82 & 0.155 \\
\hline 97 & {$[45]$} & 0 & 6.382979 & 0.066577 & 0.73 & 0.4801 \\
\hline 98 & {$[45]$} & 0 & 4.255319 & 0.066577 & 0.73 & 0.6481 \\
\hline 99 & {$[45]$} & 0 & 2.12766 & 0.066577 & 0.73 & $0.9762]$ \\
\hline 100 & {$[45]$} & 0.493462 & 4.444444 & 0.168462 & 0.94 & 0.9069 \\
\hline 101 & {$[45]$} & 0.493462 & 5.740741 & 0.168462 & 0.94 & 0.8053 \\
\hline 102 & {$[45]$} & 0.493462 & 9.481481 & 0.168462 & 0.94 & 0.5376 \\
\hline 103 & {$[45]$} & 0.986923 & 3.037037 & 0.168462 & 0.94 & 0.9953 \\
\hline 104 & {$[45]$} & 0.986923 & 4.444444 & 0.168462 & 0.94 & 0.9335 \\
\hline 105 & {$[45]$} & 0.986923 & 5.740741 & 0.168462 & 0.94 & 0.7744 \\
\hline 106 & {$[45]$} & 0.986923 & 8.962963 & 0.168462 & 0.94 & 0.5621 \\
\hline 107 & {$[45]$} & 1.480385 & 4.555556 & 0.168462 & 0.94 & 0.7937 \\
\hline 108 & {$[45]$} & 1.480385 & 5.740741 & 0.168462 & 0.94 & 0.7434 \\
\hline 109 & {$[45]$} & 1.480385 & 9.259259 & 0.168462 & 0.94 & 0.5313 \\
\hline 110 & {$[45]$} & 1.973847 & 2.962963 & 0.168462 & 0.94 & 1 \\
\hline 111 & {$[45]$} & 1.973847 & 5.592593 & 0.168462 & 0.94 & 0.7737 \\
\hline 112 & {$[45]$} & 1.973847 & 8.962963 & 0.168462 & 0.94 & 0.5687 \\
\hline 113 & {$[45]$} & 2.467308 & 2.962963 & 0.168462 & 0.94 & 0.8802 \\
\hline 114 & {$[45]$} & 2.467308 & 4.444444 & 0.168462 & 0.94 & 0.8268 \\
\hline 115 & {$[45]$} & 2.467308 & 5.481481 & 0.168462 & 0.94 & 0.811 \\
\hline 116 & {$[45]$} & 2.467308 & 9.074074 & 0.168462 & 0.94 & 0.5748 \\
\hline 117 & {$[45]$} & 2.96077 & 2.814815 & 0.168462 & 0.94 & 0.9265 \\
\hline 118 & {$[45]$} & 2.96077 & 4.444444 & 0.168462 & 0.94 & 0.8002 \\
\hline 119 & {$[45]$} & 2.96077 & 5.481481 & 0.168462 & 0.94 & 0.8218 \\
\hline 120 & {$[45]$} & 2.96077 & 8.814815 & 0.168462 & 0.94 & 0.5715 \\
\hline
\end{tabular}

Table 2. The range of datasets.

\begin{tabular}{cccc}
\hline \multicolumn{2}{c}{ Parameter } & Minimum & Maximum \\
\hline \multirow{4}{*}{ Input } & $\frac{P}{P_{0}}$ & 0.0000 & 3.9477 \\
& $\frac{S}{h_{b}}$ & 0.0000 & 300.0000 \\
& $\frac{\theta_{r}}{\theta_{s}}$ & 0.0000 & 0.7137 \\
& $\lambda$ & 0.1900 & 11.8200 \\
\multirow{4}{*}{ Output } & $\chi$ & 0.0910 & 1.0000 \\
\hline
\end{tabular}

accuracy and optimize the clustering and training time consumption, finding the appropriate number of fuzzy rules is very important. A number of methods have been recommended to gain the optimum number of clusters such as the cluster validity measure [35,50,51], the compatible cluster merging $[52,53]$, and the trialand-error method by minimizing the prediction er- ror [54]. In addition, the overfitting may occur in the training process if the number of training epochs is not selected in the appropriated range, leading to misleading results [55].

The accuracy and performance evaluation of SCFIS and FCM-FIS models should be verified by testing datasets for their first appearance in ANFIS models. Performance indices such as the determination coefficient $\left(R^{2}\right)$, the Mean Absolute Error (MAE), and the Root Mean Square Error (RMSE) were calculated to assess the accuracy of SC-FIS and FCM-FIS models. These performance measures are presented in Eqs. (28) to $(30)$ :

$$
R^{2}=\frac{\sum_{i=1}^{n} X_{i m} X_{i p}}{\sqrt{\sum_{i=1}^{n} X_{i m}{ }^{2} \sum_{i=1}^{n} X_{i p}^{2}}}
$$




$$
\begin{aligned}
& R M S E=\sqrt{\frac{\sum_{i=1}^{n}\left(\mathrm{X}_{i m}-\mathrm{X}_{i p}\right)^{2}}{n}}, \\
& M A E=\frac{\sum_{i=1}^{n}\left|\mathrm{X}_{i m}-\mathrm{X}_{i p}\right|}{n},
\end{aligned}
$$

where $n$ is the number of data in each dataset, $X_{i m}$ is the measured value, and $X_{i p}$ is the predicted value.

In the following sections, to predict parameter $\chi$, the process of finding the optimum number of training epochs and fuzzy rules along with some other key features of the two ANFIS models is presented.

\subsection{SC-FIS model}

The initial FIS of SC-FIS model was created by SC. In order to determine the best epoch of training ANFIS, some FIS models were created by SC with a range of $r_{a}$ between 0.1 and 1 and the random choice of Training, Validation, and Testing (TVT) datasets. Other SC chosen parameters include $\eta=1.25, \bar{\varepsilon}=0.5$, and $\underline{\varepsilon}=0.15$. Then, the number of epochs for training was set to 1000. In each epoch, the RMSE was calculated for training and validation sets as an error tolerance. When the designated epoch number was reached, the

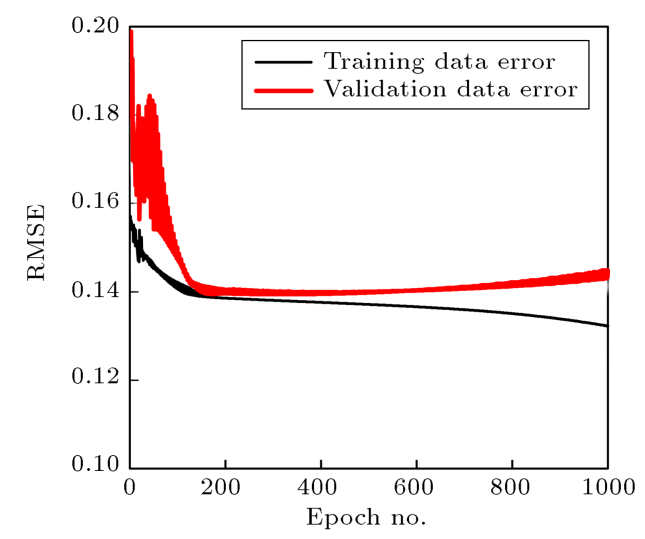

(a)

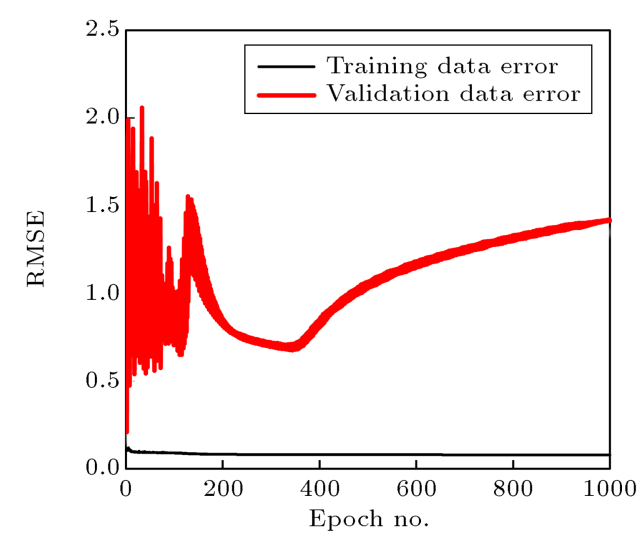

(c) error tolerance was plotted. Some error tolerance plots were tried in different $r_{a}$ and random datasets. Figure 4 represents the plots of four examples of error tolerance for training and validation datasets. In Figure 4(a) and (b), after about 200 epochs, the error tolerance nearly gets constant, and no considerable decrease occurs. As shown in Figure 4(c), overfitting occurs after about 400 epochs. Figure $4(d)$ is an example of when the overfitting occurs in the initial epochs and the training is not good; then, in order to prevent overfitting, the TVT datasets can be randomly chosen, and the appropriate dataset is obtained to train the model. The best number of epochs for the training process is about 200 to 300 epochs, and it is normally enough for the training process.

The value of $r_{a}$ strongly affects the number of fuzzy clusters. As $r_{a}$ increases, the fuzzy rules decrease, and vice versa. Therefore, finding the best $r_{a}$ is very important for achieving a suitable solution to the problem. To get the best $r_{a}$ in SC-FIS model, the trialand-error method is used and the code is written in MATLAB software with the following conditions.

The TVT datasets were created randomly, and the range of $r_{a}$ was chosen between 0.1 and 1 . This

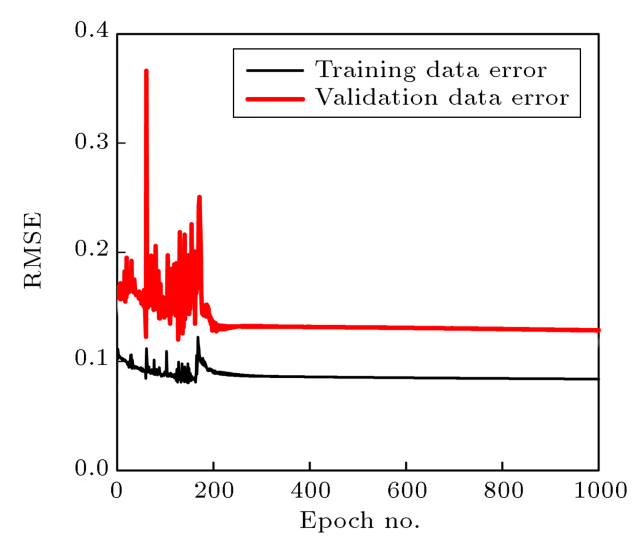

(b)

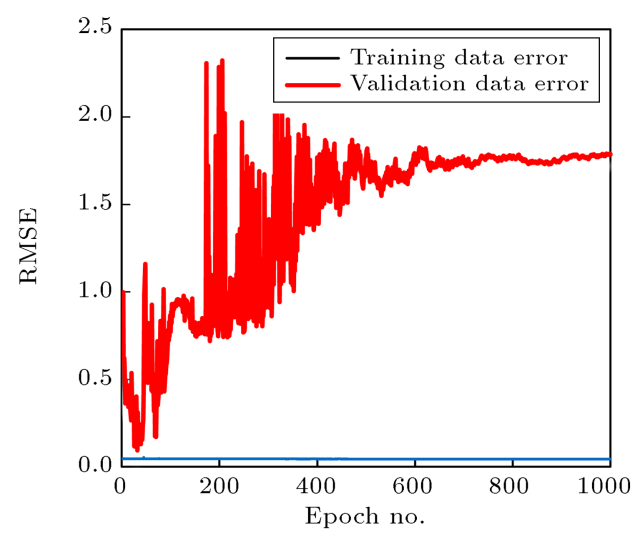

(d)

Figure 4. Examples of the number of epochs versus error tolerance for training and validation datasets for the fuzzy model with the initial SC-FIS model. 
range was divided into 25 intervals. The initial FIS was created at each $r_{a}$ interval, and the number of clusters corresponding to $r_{a}$ intervals was determined. Then, the initial FIS was trained with an epoch equal to 300 . For each $r_{a}, R^{2}$ value was calculated for the TVT datasets. The above steps were repeated for the specified iterations. For each $r_{a}$ interval, the average of all $R^{2} s$ and cluster numbers was calculated and obtained in each iteration corresponding to the $r_{a}$ interval. The $r_{a}$ versus average $R^{2}$ is plotted in Figure 5. As can be seen in Figure $5, R^{2}$ has the highest value when $r_{a}$ is determined to be between 0.5 and 0.65. Figure 6 shows the $r_{a}$ versus the number of clusters. As can be seen in this figure, an increase in $r_{a}$ decreases the number of clusters. Figure 7 shows the number of clusters versus $R^{2}$ for SC. In the range of $r_{a}=0.5 \sim 0.65$, there are 5 to 10 clusters.

After finding a proper range of $r_{a}$, the SC-FIS model was developed to predict $\chi$ value. For this purpose, SC parameters were chosen as follows: $\eta=$ $1.25, \bar{\varepsilon}=0.5$, and $\underline{\varepsilon}=0.15, r_{a}=0.5 \sim 0.65$ by

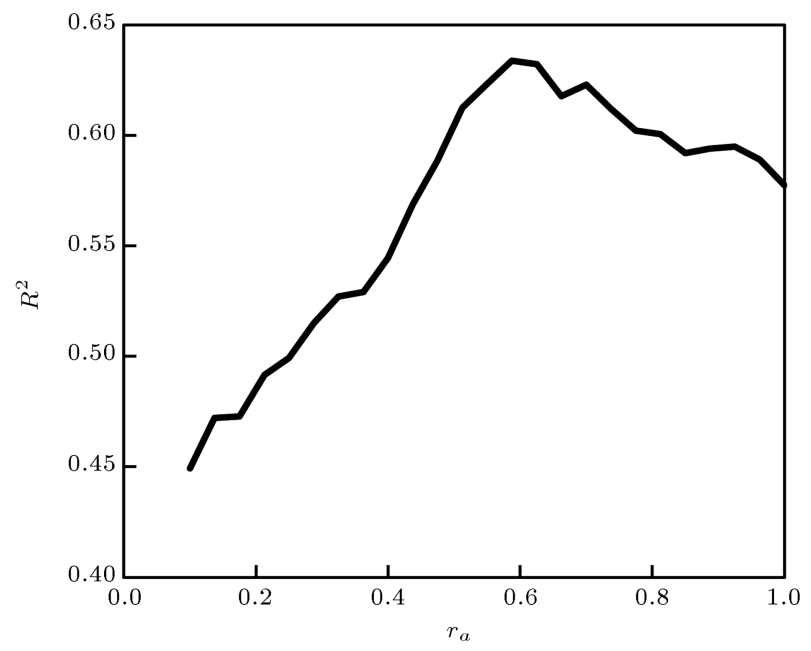

Figure 5. Variable $r_{a}$ versus average $R^{2}$.

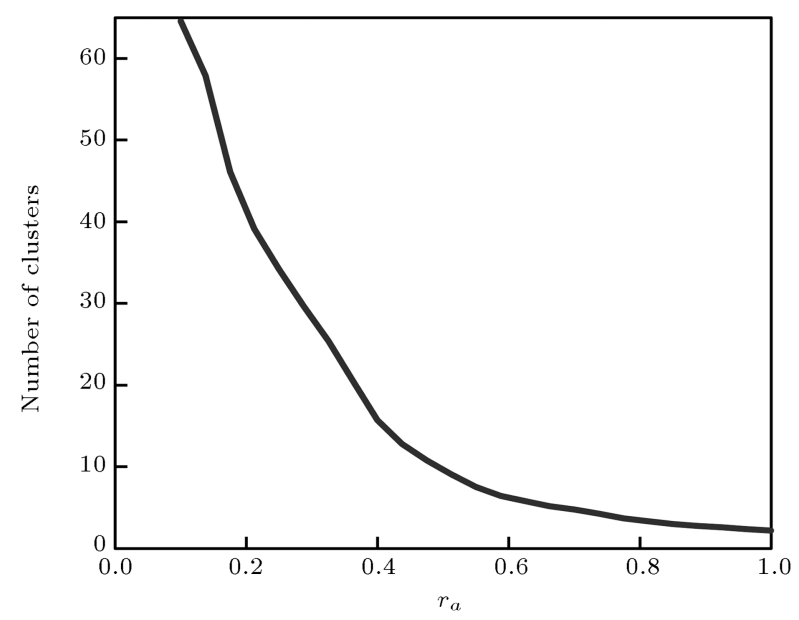

Figure 6. Variable $r_{a}$ versus the number of clusters.

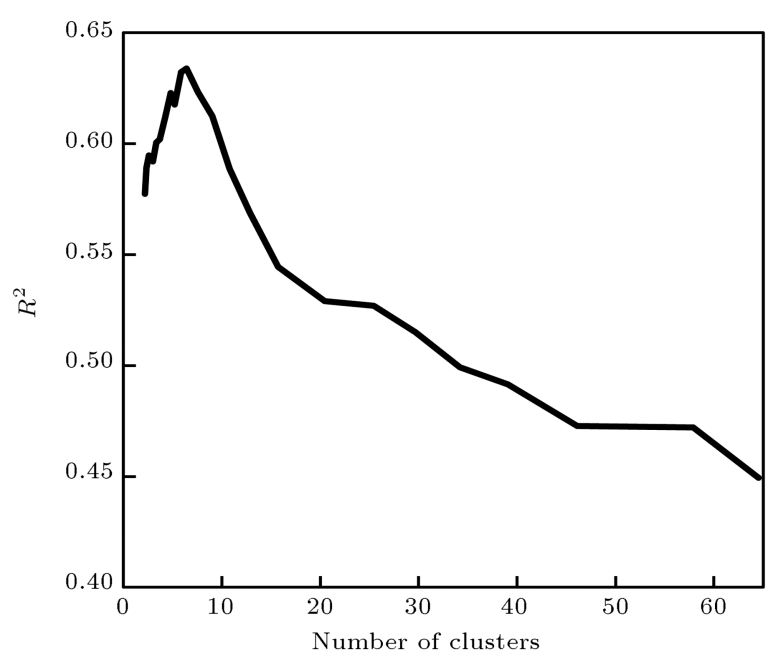

Figure 7. Number of clusters versus $R^{2}$.

dividing into 25 intervals, epoch $=300$, and initial $R^{2}$ $=0$. The TVT datasets were randomly created. For each $r_{a}$ interval, the initial FIS was also created and trained by ANFIS; accordingly, the value of $R^{2}$ was calculated for the TVT and the total dataset. Then, the new $R^{2}$ for the total dataset was compared to the previous $R^{2}$. If $R^{2}$ was greater than the previous $R^{2}$, the ANFIS was stored and, then, continued at the next $r_{a}$ interval. After finishing all $r_{a}$ intervals, the next iteration and the other TVT dataset were randomly selected, and the above process was repeated. This process continued until the model was not able to find any greater $R^{2}$. The best $R^{2}$ for the SCFIS model was found in $r_{a}=0.55$ with 9 rules. The membership functions before and after training are shown in Figure 8 for the SC-FIS model. The comparison results of the predicted and measured $\chi$ values for the TVT datasets are shown in Figure 9(a) to $(\mathrm{c})$. The measured $\chi$ versus predicted $\chi$ values and, also, the line by $R^{2}=1$ for the TVT datasets are shown in Figure 10(a) to (c). As can be seen in these figures, there is good agreement between measured and predicted $\chi$ values obtained by the SC-FIS model in the training, validation, and testing datasets.

\subsection{FCM-FIS model}

The initial FIS of the FCM-FIS model was created by the FCM clustering. In order to assign a proper epoch according to the random TVT datasets, a number of FIS models were created by the FCM clustering, and the number of epochs for training was adjusted to 1000. Figure 11 shows four error tolerance examples in epochs for training and validation datasets by the FCM clustering and initial FIS. As can be seen in Figure 11(a) to (c), after about 100 to 200 epochs, the error tolerance reaches a plateau. This situation was also considered in many other error tolerance figures for various ANFIS training models. Figure 11(d) is an 

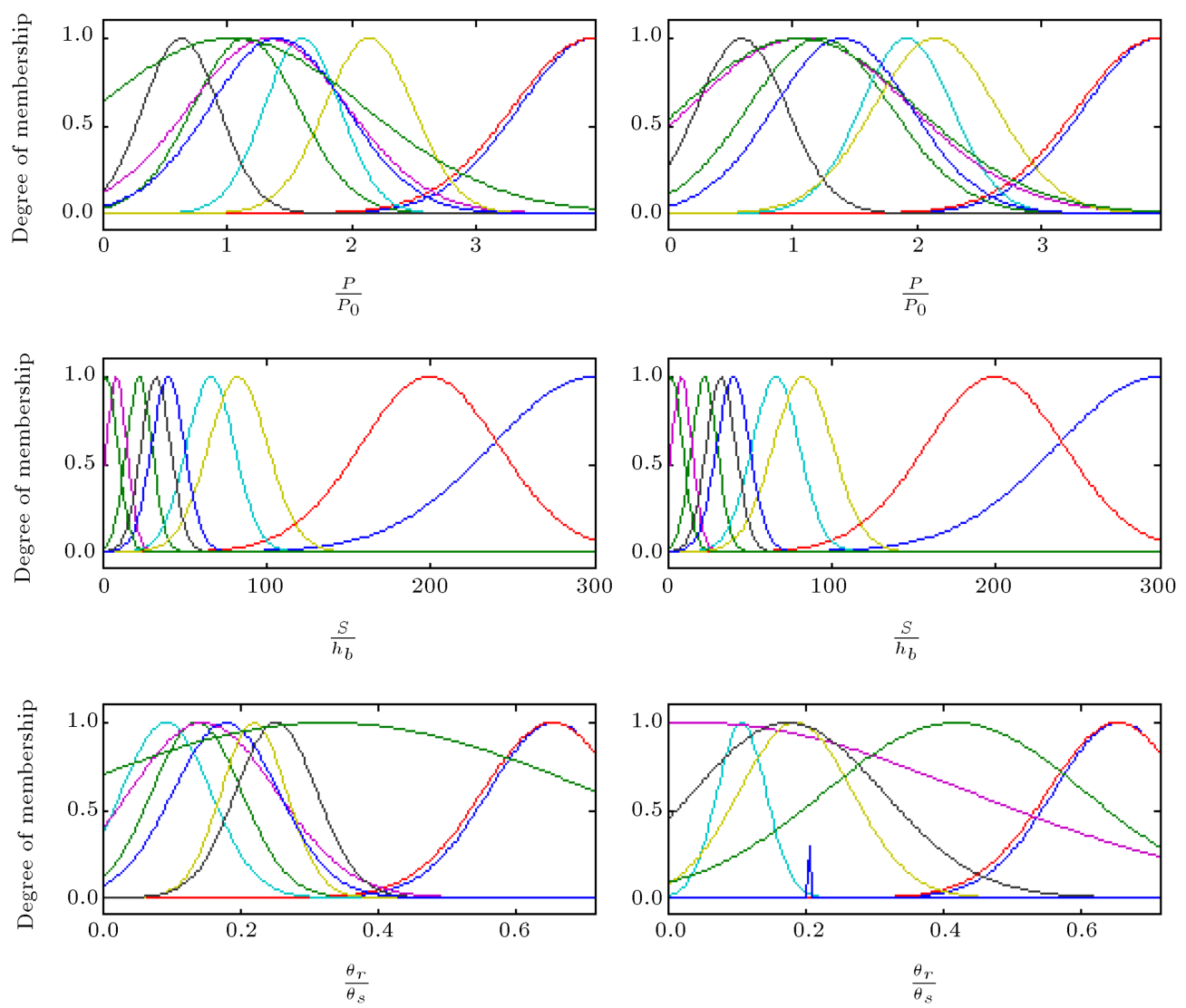

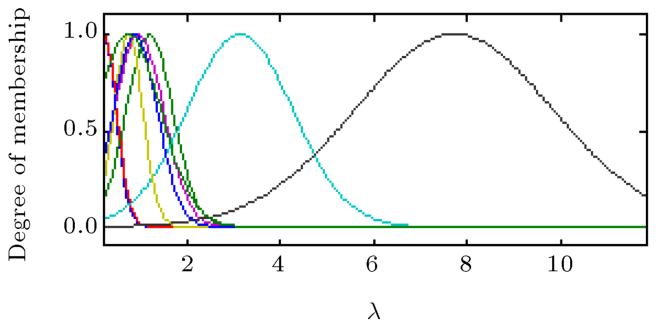

(a)

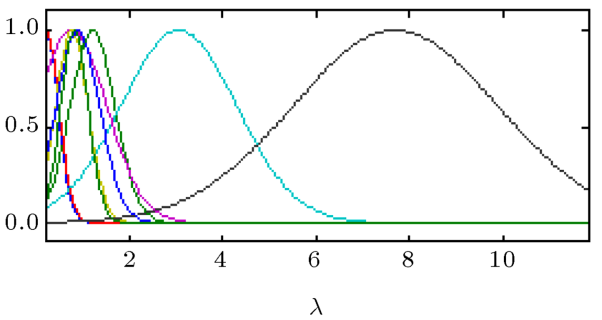

(b)

Figure 8. The membership functions: (a) Before and (b) after training for SC-FIS model.

example of overfitting (after about 200 epochs). The number of epochs for the model by the initial FCM-FIS is assumed as 200 epochs.

In order to determine the best number of clusters, a MATLAB code was written and used. In this code, for each iteration, the number of clusters was taken to be between 2 and 50, and the data points for the TVT datasets were selected randomly from the total dataset. Then, for each number of cluster intervals, the initial FIS was created and trained by ANFIS with 200 epochs. $R^{2}$ was calculated according to each number of cluster intervals. After reaching the last interval of clusters, the next iteration should start; then, the TVT datasets are selected randomly, and these steps are repeated. Following the termination of the desired iteration of selecting random datasets, the average value of $R^{2}$ was calculated for each cluster interval. Then, the number of cluster intervals was plotted versus average $R^{2}$. Next, the range of the best number of clusters was selected according to the highest $R^{2}$. Figure 12 shows the number of clusters versus $R^{2}$. According to Figure $12, R^{2}$ has the highest value in 7 to 10 clusters.

To develop the FCM-FIS model, a MATLAB code was written. The number of clusters was chosen to be between 7 and 10. For the first iteration, it was assumed that $R^{2}=0$ and the epoch was taken to be 200. For each cluster interval, TVT datasets were created randomly, and the initial FIS was created by the FCM clustering and trained by ANFIS. The value of $R^{2}$ was calculated according to TVT and total dataset. Then, the new $R^{2}$ for the total dataset was 


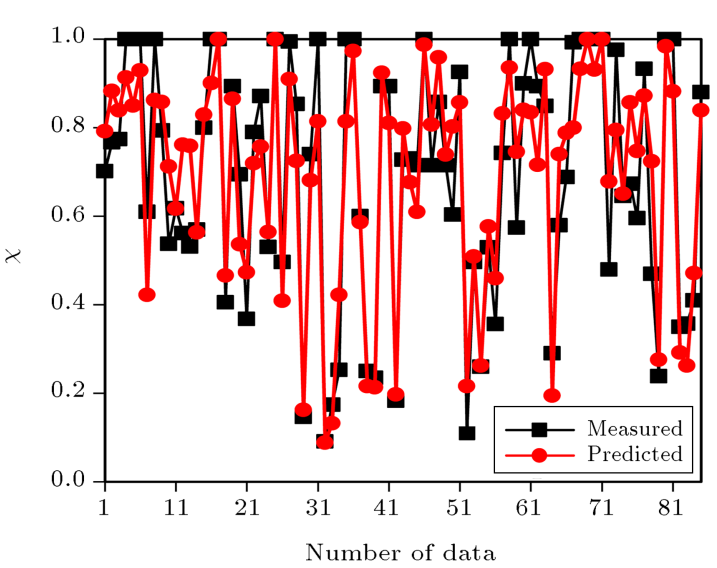

(a)

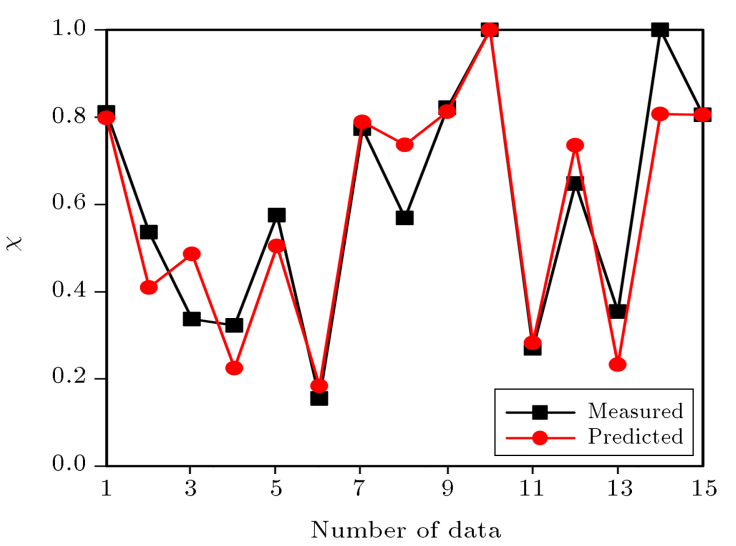

(b)

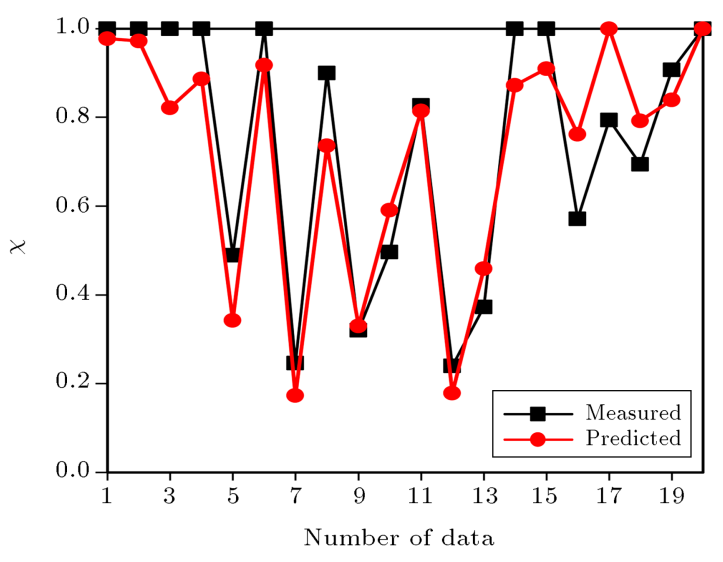

(c)

Figure 9. Comparison of the predicted $\chi$ values by SC-FIS model for (a) training, (b) validation, and (c) testing datasets.

compared with the previous $R^{2}$. If $R^{2}$ was greater than the previous $R^{2}$, the ANFIS was stored and, then, continued at the next number of cluster intervals. After completing all cluster intervals, other TVT datasets were randomly selected, and the process above was repeated. This process continued until the model failed to find any greater $R^{2}$. The FCM-FIS model found the best $R^{2}$ in 9 clusters. The membership

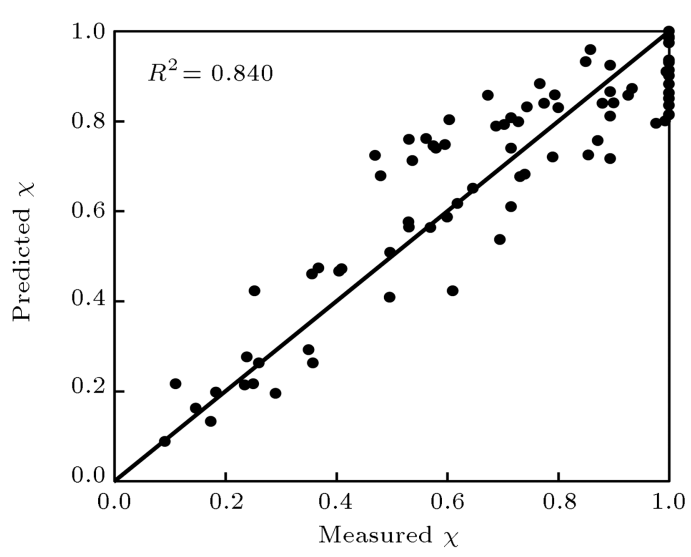

(a)

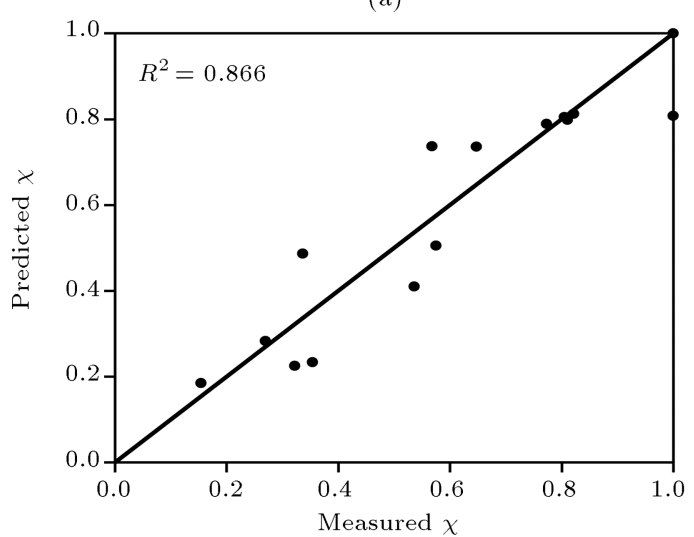

(b)

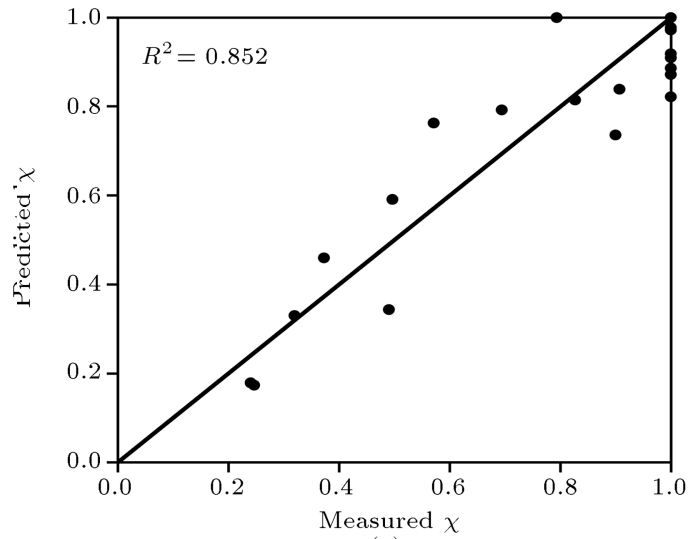

(c)

Figure 10. The measured $\chi$ versus predicted $\chi$ values by SC-FIS model and, also, the line by $R^{2}=1$ for (a) training, (b) validation, and (c) testing dataset.

functions before and after training of the FCM-FIS model are shown in Figure 13. A comparison of the predicted and measured $\chi$ values for the TVT datasets is represented in Figure $14(\mathrm{a})$ to $(\mathrm{c})$. The measured $\chi$ versus the predicted $\chi$ values and the line by $R^{2}=1$ for training, validation, and testing datasets are plotted in Figure $15(\mathrm{a})$ to $(\mathrm{c})$, respectively.

\section{Sensitivity analysis}

To determine the relative effect of the input parameters 


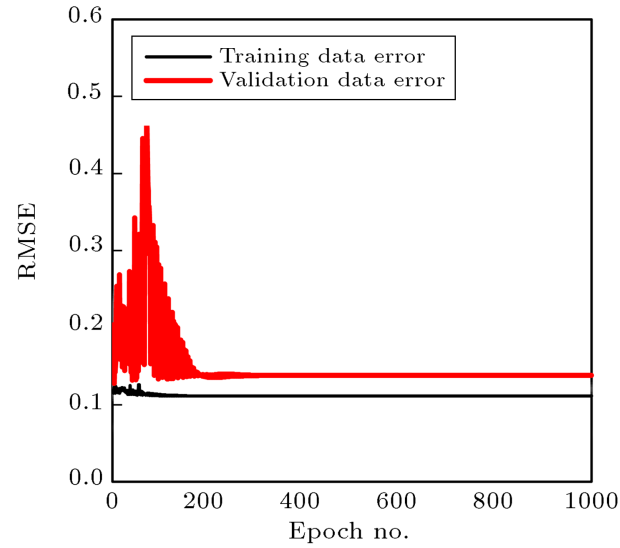

(a)

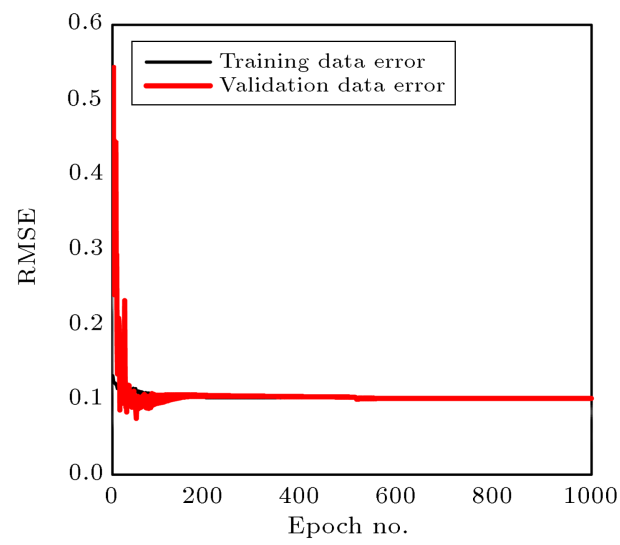

(c)

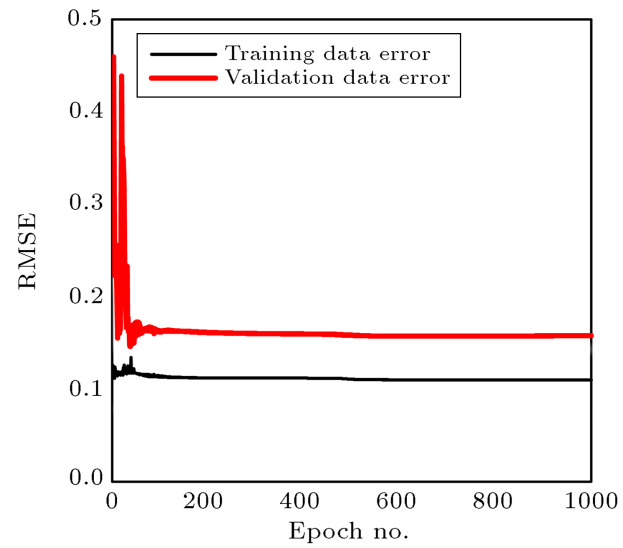

(b)

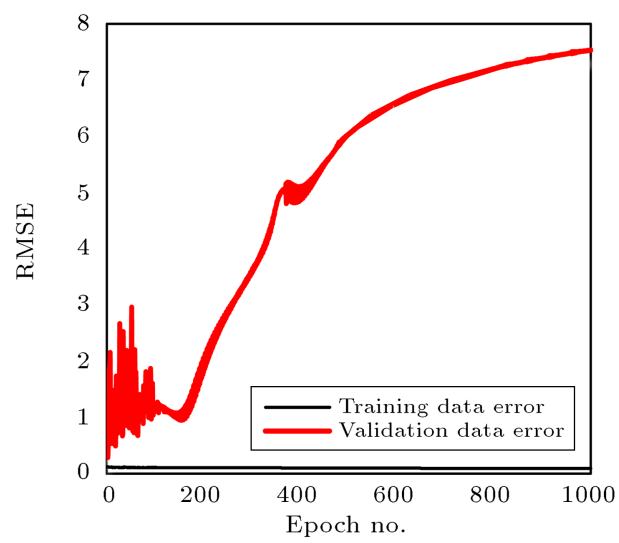

(d)

Figure 11. Four examples of the most observed samples of the number of epochs versus error tolerance for training and validation datasets for the fuzzy model with the initial FCM-FIS model.

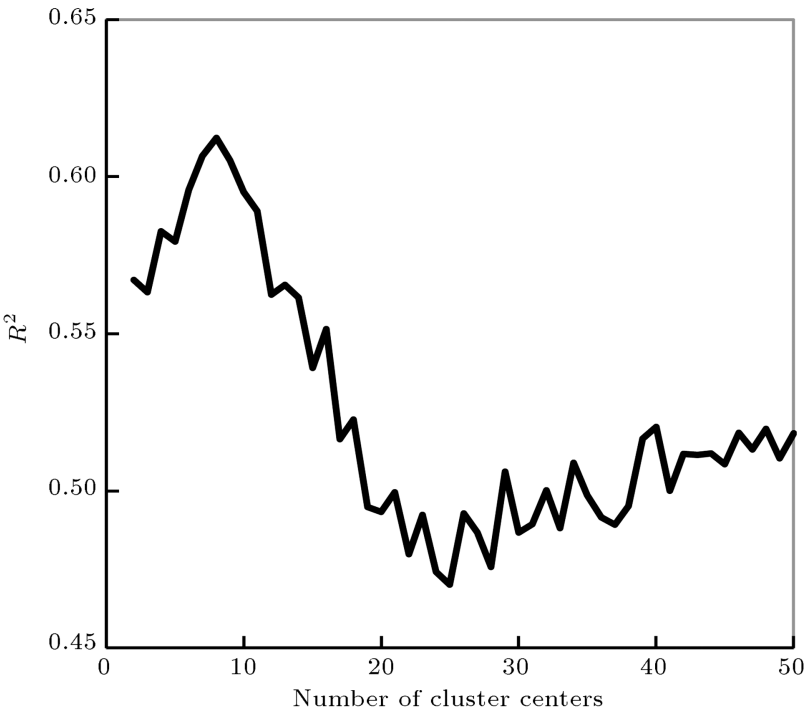

Figure 12. Number of clusters versus $R^{2}$.

on $\chi$ value, sensitivity analysis was conducted for all major input parameters. The Cosine Amplitude Method (CAM) [56] was used to obtain the relationship between inputs and outputs. Hence, matrix $Z$ that contains the data points is considered as follows:

$$
\left\{\begin{array}{l}
Z=\left\{Z_{1}, Z_{2}, \ldots, Z_{n}\right\} \\
Z_{k}=\left\{z_{I k} z_{O k}\right\} k=1,2, \ldots, n \\
z_{I k}=\left\{z_{k 1}, z_{k 2}, \ldots, z_{k m}\right\} \\
z_{O k}=\left\{z_{k 1}, z_{k 2}, \ldots, z_{k o}\right\}
\end{array}\right.
$$

where $n$ is the number of data points; for each data point, $z_{I k}$ is the input vector with lengths of $m, Z_{O k}$ is the output vector with a length of $o$, and $Z_{k}$ is the vector with a length of $m+o$. In other words, the dataset can be represented by $n$ data points in $m+o$ dimensional space.

$r_{i j}$ is considered as the relative influence of each input on each output:

$$
\begin{gathered}
r_{i j}=\frac{\sum_{k=1}^{n} z_{k i} z_{k j}}{\sqrt{\sum_{k=1}^{n} z_{k i}^{2} \sum_{k=1}^{n} z_{k j}^{2}}} \\
i=1,2, \ldots, m \quad j=1,2, \ldots, o .
\end{gathered}
$$

In this paper, $m=4$ (for input measured parameters: $\frac{P}{P_{0}}, \frac{S}{h_{b}}, \frac{\theta_{r}}{\theta_{s}}$, and $\lambda$ ) and $o=1$ (for output 

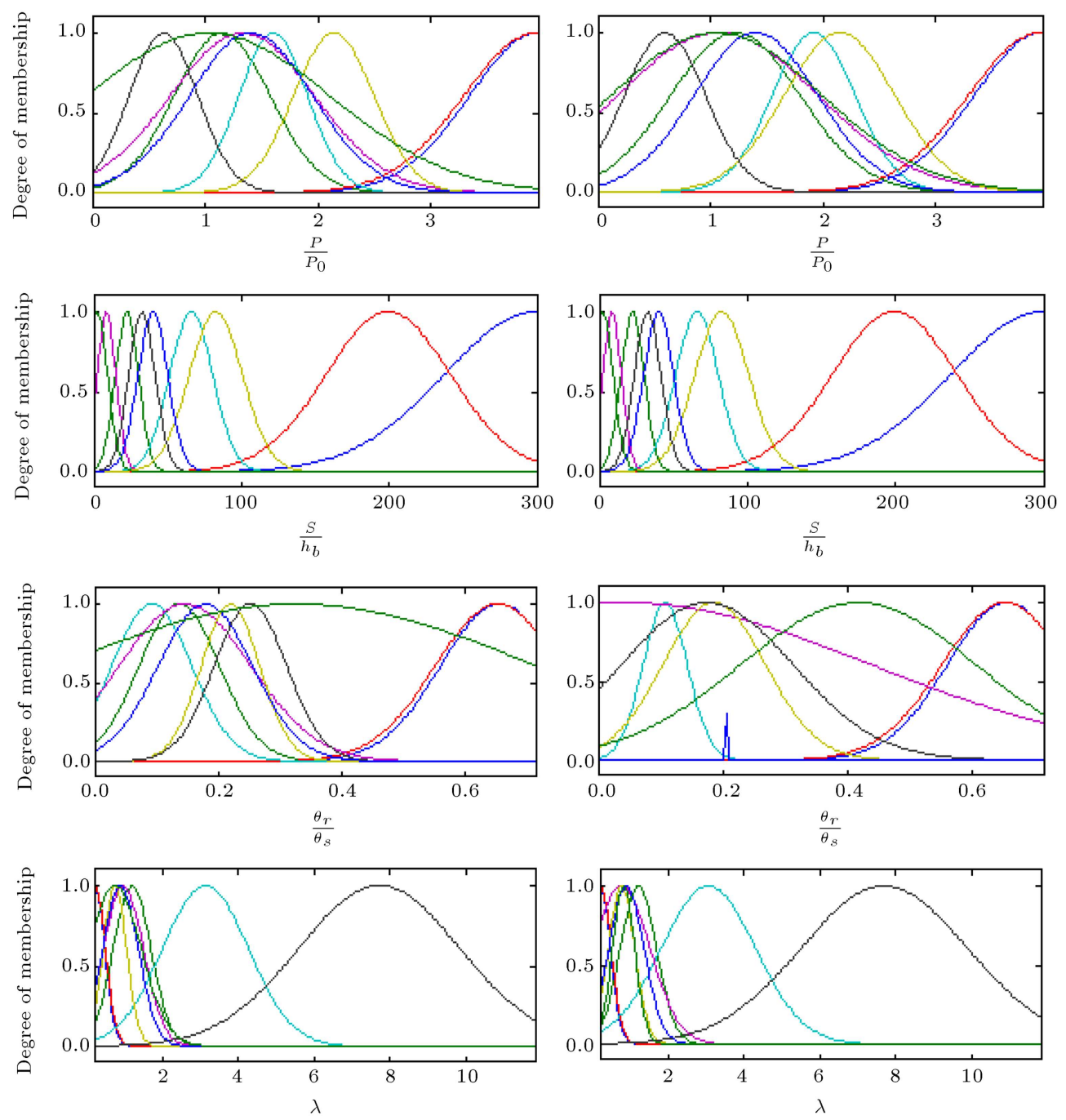

(a)

(b)

Figure 13. The membership functions: (a) Before and (b) after training for FCM-FIS model.

measured parameter: $\chi$ ). The sensitivity analysis of input parameters is represented in Figure 16. As can be seen, $\frac{P}{P_{0}}$ and $\frac{\theta_{r}}{\theta_{s}}$ are the most influential parameters, whereas $\frac{S}{h_{b}}$ has the least effect on $\chi$ value.

\section{Results and discussion}

The values of $R^{2}$, RMSE, and MAE for SC-FIS and FCM-FIS models are given in Table 3. The total dataset in Table 3 consists of the TVT datasets. Table 3 also contains the values of $R^{2}$ for the GEP model, as proposed by Johari et al. [15]. Figure 17 shows the charts of $R^{2}$, RMSE, and MAE with respect to the developed ANFIS models and GEP $R^{2}$ s. As shown in Table 3 and Figure 17, both ANFIS models were compared with the GEP model and presented good predictions of $\chi$ value, while the FCM-FIS model yielded a relatively better prediction than that of the SC-FIS model. $R^{2}$ value for the FCM-FIS model was
Table 3. The values of performance indices for the two proposed models and $R^{2}$ for the GEP model developed by Johari et al. [15].

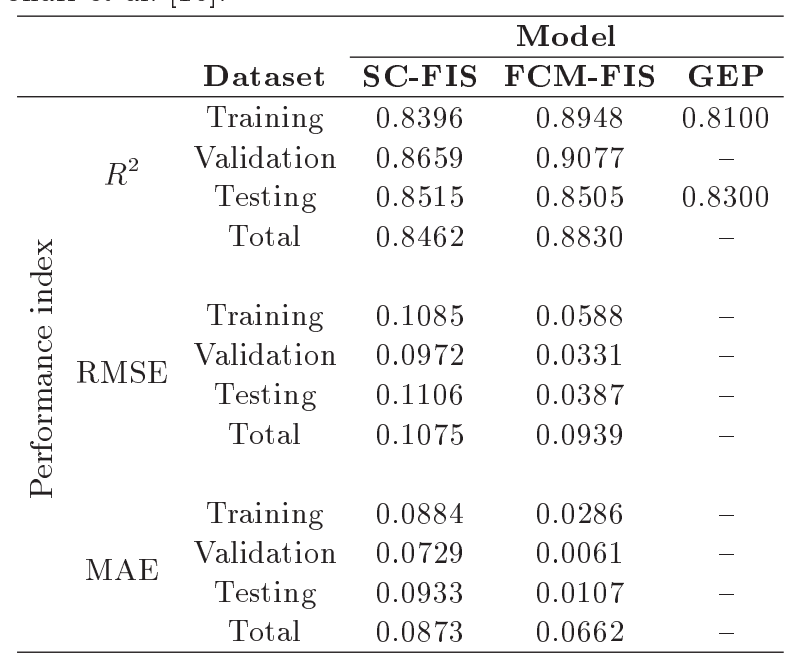




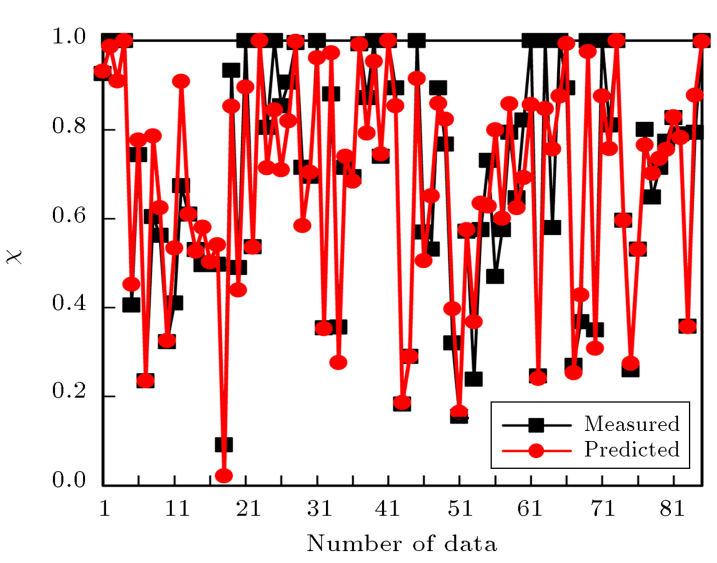

(a)

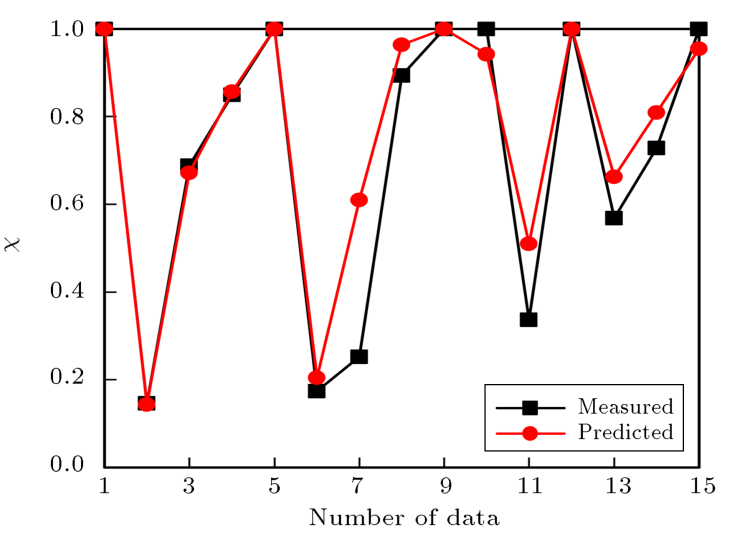

(b)

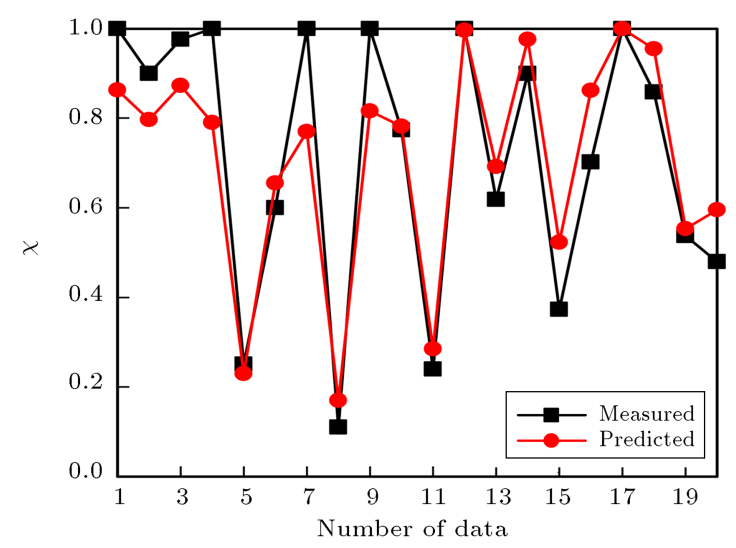

(c)

Figure 14. Comparison of the predicted $\chi$ and the measured $\chi$ values by FCM-FIS model for (a) training, (b) validation, and (c) testing datasets.

greater than and closer to 1 in comparison with the SCFIS model. According to Smith [57], if the proposed model gives $R^{2}>0.8$, there will generally be a strong correlation between measured and estimated values over all available data in the database.

The sensitivity analysis of input parameters is represented in Figure 17. This figure shows that $\frac{P}{P_{0}}$ and $\frac{\theta_{r}}{\theta_{s}}$ are the most effective parameters, and $\frac{S}{h_{b}}$ has the least effect on $\chi$ value.

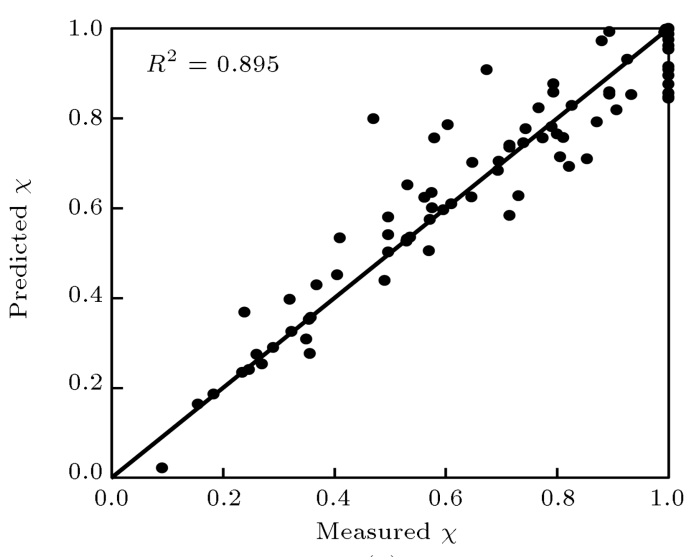

(a)

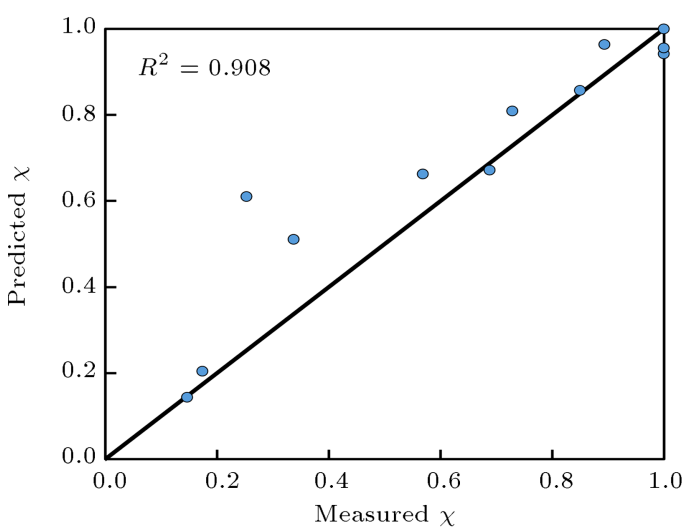

(b)

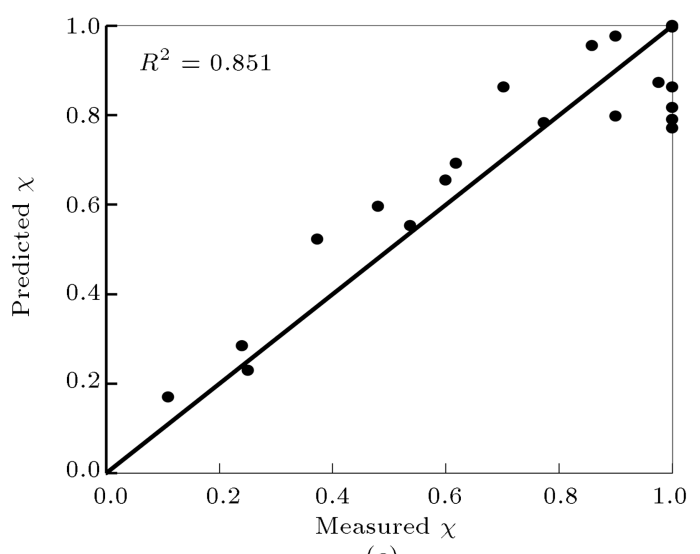

(c)

Figure 15. The measured $\chi$ versus predicted $\chi$ values by FCM-FIS model and, also, the line by $R^{2}=1$ for (a) training, (b) validation, and (c) testing datasets.

\section{Conclusions}

The value of the effective stress parameter, $\chi$, is important while dealing with unsaturated soil mechanics. In this paper, two ANFIS models were developed to predict the most effective stress parameter. These models include SC-FIS and FCM-FIS ones, and the initial FIS of these models was created by Subtractive Clustering (SC) and Fuzzy c-means (FCM) clustering, respectively. The datasets used for developing the two ANFIS models were collected from the literature and, 


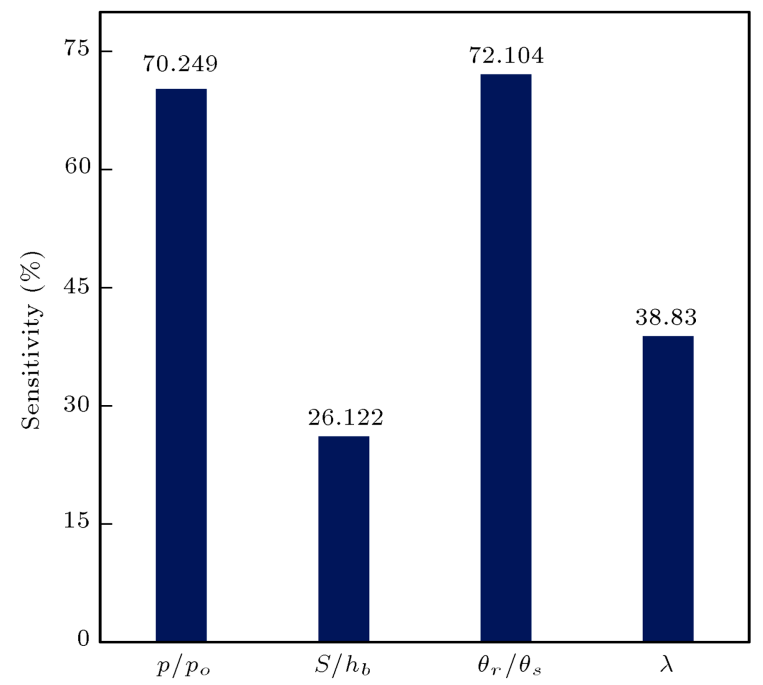

Figure 16. Sensitivity analysis of input parameters of $\chi$ models.
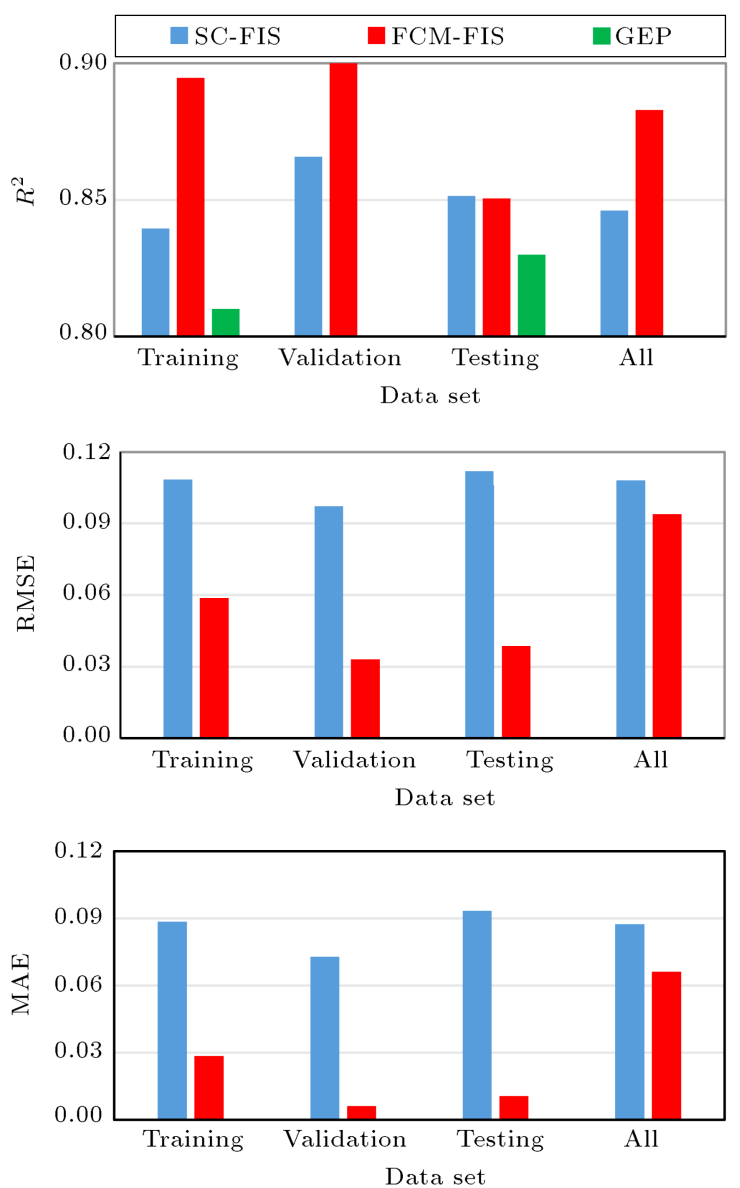

Figure 17. The charts of $R^{2}$, RMSE, and MAE for the developed ANFIS models.

also, the results of 120 triaxial, shear, pressure plate and filter paper tests. The key inputs of the model included the dimensionless suction parameter $\left(\frac{S}{h_{b}}\right)$, the dimensionless confining pressure parameter $\left(\frac{P}{P_{0}}\right)$, the dimensionless volumetric water content parameter $\left(\frac{\theta_{r}}{\theta_{s}}\right)$, and the soil water characteristic curve fitting parameter $(\lambda)$. In order to develop the models with the highest generalization capability, $r_{a}$ in SC, the number of the cluster in the FCM clustering, and the ANFIS training epochs were optimized by the trialand-error method. Performance indices, such as $R^{2}$, MAE, and RMSE, were calculated to compare the efficiency of ANFIS models. The results of ANFIS models were compared with the Gene Expression Programming (GEP) model, presented by Johari et al. [15]. Sensitivity analysis was carried out to determine the effect of model inputs $\left(\frac{S}{h_{b}}, \frac{P}{P_{0}}, \frac{\theta_{r}}{\theta_{s}}\right.$ and $\left.\lambda\right)$ on the model output $(\chi)$. The following concluding remarks can be drawn from the results of this study:

1. Both ANFIS models have shown the capability to reasonably predict parameter $\chi$ in the ANFIS modeling;

2. $R^{2}$ s of SC-FIS and FCM-FIS models for the total datasets were 0.8462 and 0.8830 , respectively. These values showed that the ANFIS models were able to be used to predict $\chi$ value;

3. The RMSE of SC-FIS and FCM-FIS models for the total dataset were 0.1075 and 0.0939 , respectively, which were small and acceptable values for the prediction of $\chi$;

4. FCM-FIS model provided relatively better results than SC-FIS model did;

5. ANFIS model's results were better than the GEP model results, as presented by Johari et al. [15];

6. Sensitivity analysis results showed that $\frac{P}{P_{0}}$ and $\frac{\theta_{r}}{\theta_{s}}$ were the most sensitive parameters, while $\frac{S}{h_{b}}$ had the least effect on $\chi$ value.

In summary, using the proposed methods would decrease the number of laboratory tests; therefore, considerable cost and time could be saved.

\section{References}

1. Fredlund, D.G. and Morgenstern, N.R. "Stress state variables for unsaturated soils", Journal of Geotechnical and Geoenvironmental Engineering, 103(5), pp. 447-466 (1977).

2. Terzaghi, K., Theoretical Soil Mechanics, London: Chapman and Hall Limited: John Wiley and Sons, Inc. New York (1943).

3. Croney, D., Coleman, J.D., and Black, W.P.M., Studies of the Movement and Distribution of Water in Soil in Relation to Highway Design and Performance, Transport and Road Research Laboratory (1958).

4. Bishop, A.W. "The principle of effective stress", published in Teknisk Ukeblad, 106(39), pp. 859-863 (1959). 
5. Richards, B. "The significance of moisture flow and equilibria in unsaturated soils in relation to the design of engineering structures built on shallow foundations in Australia", In: Symposium on Permeability and Capillarity, ASTM, Atlantic City, NJ. Richards, L.A (1966).

6. Aitchison, G.D. "Moisture equilibria and moisture changes in soils beneath covered areas: a symposium in print", Engineering Concepts of Moisture Equilibria and Moisture Changes in Soils, Statement of the review panel, G.D. Aitchison, Ed., 8(1), pp. 7-21 (1965).

7. Khalili, N. and Khabbaz, M. "A unique relationship of chi for the determination of the shear strength of unsaturated soils", Geotechnique, 48(5), pp. 681-687 (1998).

8. Lu, N. and Likos, W.J., Unsaturated Soil Mechanics, John Wiley \& Sons (2004).

9. Fredlund, D., Morgenstern, N., and Widger, R. "The shear strength of unsaturated soils", Canadian Geotechnical Journal, 15(3), pp. 313-321 (1978).

10. Escario, V. and Saez, J. "The shear strength of partly saturated soils", Geotechnique, 36(3), pp. 453-456 (1986).

11. Vanapalli, S., Fredlund, D., Pufahl, D., and Clifton, A. "Model for the prediction of shear strength with respect to soil suction", Canadian Geotechnical Journal, 33(3), pp. 379-392 (1996).

12. Fredlund, D.G., Xing, A., Fredlund, M.D., and Barbour, S. "The relationship of the unsaturated soil shear to the soil-water characteristic curve", Canadian Geotechnical Journal, 33(3), pp. 440-448 (1996).

13. Kayadelen, C. "Estimation of effective stress parameter of unsaturated soils by using artificial neural networks", International Journal for Numerical and Analytical Methods in Geomechanics, 32(9), pp. 10871106 (2008).

14. Ajdari, M., Habibagahi, G., and Ghahramani, A. "Predicting effective stress parameter of unsaturated soils using neural networks", Computers and Geotechnics, 40, pp. 89-96 (2012).

15. Johari, A., Nakhaee, M., and Habibagahi, G. "Prediction of unsaturated soils effective stress parameter using gene expression programming", Scientia Iranica, 20(5), pp. 1433-1444 (2013).

16. Jang, J.-S. "ANFIS: adaptive-network-based fuzzy inference system", Systems, Man and Cybernetics, IEEE Transactions on, 23(3), pp. 665-685 (1993).

17. Kar, S., Das, S., and Ghosh, P.K. "Applications of neuro fuzzy systems: A brief review and future outline", Applied Soft Computing, 15, pp. 243-259 (2014).

18. Gokceoglu, C., Yesilnacar, E., Sonmez, H., and Kayabasi, A. "A neuro-fuzzy model for modulus of deformation of jointed rock masses", Computers and Geotechnics, 31(5), pp. 375-383 (2004).
19. Kalkan, E., Akbulut, S., Tortum, A., and Celik, S. "Prediction of the unconfined compressive strength of compacted granular soils by using inference systems", Environmental Geology, 58(7), pp. 1429-1440 (2009).

20. Kayadelen, C., Taşkıran, T., Günaydın, O., and Fener, M. "Adaptive neuro-fuzzy modeling for the swelling potential of compacted soils", Environmental Earth Sciences, 59(1), pp. 109-115 (2009).

21. Cobaner, M. "Evapotranspiration estimation by two different neuro-fuzzy inference systems", Journal of Hydrology, 398(3), pp. 292-302 (2011).

22. Sezer, A., Göktepe, A., and Altun, S. "Adaptive neurofuzzy approach for sand permeability estimation", Environ Eng Manag J, 9(2), pp. 231-238 (2010).

23. Ikizler, S.B., Vekli, M., Dogan, E., Aytekin, M., and Kocabas, F. "Prediction of swelling pressures of expansive soils using soft computing methods", Neural Computing and Applications, 24(2), pp. 473485 (2012).

24. Doostmohammadi, R. "Cyclic swelling estimation of mudstone using adaptive network-based fuzzy inference system", Middle-East Journal of Scientific Research, 11(4), pp. 517-524 (2012).

25. Cabalar, A.F., Cevik, A., and Gokceoglu, C. "Some applications of adaptive neuro-fuzzy inference system (ANFIS) in geotechnical engineering", Computers and Geotechnics, 40, pp. 14-33 (2012).

26. Zoveidavianpoor, M. "A comparative study of artificial neural network and adaptive neurofuzzy inference system for prediction of compressional wave velocity", Neural Computing and Applications, 25(5), pp. 1-8 (2014).

27. Zadeh, L.A. "Fuzzy sets", Information and Control, 8(3), pp. 338-353 (1965).

28. Jang, J.S.R. and Gulley, N., Fuzzy Logic Toolbox: User's Guide, The Mathworks", Inc (2000).

29. Mamdani, E.H. and Assilian, S. "An experiment in linguistic synthesis with a fuzzy logic controller", International Journal of Man-Machine Studies, 7(1), pp. 1-13 (1975).

30. Takagi, T. and Sugeno, M. "Fuzzy identification of systems and its applications to modeling and control", Systems, Man and Cybernetics, IEEE Transactions on, 1, pp. 116-132 (1985).

31. Tsukamoto, Y. "An approach to fuzzy reasoning method", Advances in Fuzzy Set Theory and Applications, 137, p. 149 (1979).

32. Rumelhart, D.E. and McClelland, J.L., Parallel Distributed Processing, Cambridge, MA, MIT Press: IEEE (1988).

33. Hashemi Jokar, M. and Mirasi, S. "Using adaptive neuro-fuzzy inference system for modeling unsaturated soils shear strength", Soft Computing, 22(13), pp. 1-18 (2017).

34. Steinhaus, H. "Sur la division des corp materiels en parties", Bull. Acad. Polon. Sci, 4(12), pp. 801-804 (1956). 
35. Bezdek, J.C., Pattern Recognition With Fuzzy Objective Function Algorithms, Kluwer Academic Publishers (1981).

36. Chiu, S.L. "A cluster estimation method with extension to fuzzy model identification", In Proceedings of 1994 IEEE 3rd International Fuzzy Systems Conference, IEEE, pp. 1240-1245 (1994).

37. Chiu, S. "Fuzzy model identification based on cluster estimation", Journal of Intelligent and Fuzzy Systems, 2(3), pp. 267-278 (1994).

38. Yager, R.R. and Filev, D.P. "Generation of fuzzy rules by mountain clustering", Journal of Intelligent and Fuzzy Systems, 2(3), pp. 209-219 (1994).

39. Dunn, J.C. "A fuzzy relative of the ISODATA process and its use in detecting compact well-separated clusters", J. Cybernet., 3(3), pp. 32-57 (1973).

40. Rahardjo, H., Heng, O.B., and Choon, L.E. "Shear strength of a compacted residual soil from consolidated drained and constant water content triaxial tests", Canadian Geotechnical Journal, 41(3), pp. 421-436 (2004).

41. Lee, I.-M., Sung, S.-G., and Cho, G.-C. "Effect of stress state on the unsaturated shear strength of a weathered granite", Canadian Geotechnical Journal, 42(2), pp. 624-631 (2005).

42. Rassam, D.W. and Williams, D.J. "A relationship describing the shear strength of unsaturated soils", Canadian Geotechnical Journal, 36(2), pp. 363-368 (1999).

43. Khalili, N., Geiser, F., and Blight, G. "Effective stress in unsaturated soils: review with new evidence", International Journal of Geomechanics, 4(2), pp. 115126 (2004).

44. Miao, L., Liu, S., and Lai, Y. "Research of soil-water characteristics and shear strength features of Nanyang expansive soil", Engineering Geology, 65(4), pp. 261267 (2002).

45. Thu, T.M., Rahardjo, H., and Leong, E.-C. "Effects of hysteresis on shear strength envelopes from constant water content and consolidated drained triaxial tests", Unsaturated Soils, pp. 1212-1222 (2006).

46. Rampino, C., Mancuso, C., and Vinale, F. "Experimental behaviour and modelling of an unsaturated compacted soil", Canadian Geotechnical Journal, 37(4), pp. 748-763 (2000).

47. Russell, A.R. and Khalili, N. "A bounding surface plasticity model for sands exhibiting particle crushing", Canadian Geotechnical Journal, 41(6), pp. 1179-1192 (2004).

48. Russell, A. and Khalili, N. "A unified bounding surface plasticity model for unsaturated soils", International Journal for Numerical and Analytical Methods in Geomechanics, 30(3), pp. 181-212 (2006).
49. Bishop, A.W. and Blight, G.E. "Some aspects of effective stress in saturated and partially saturated soils", Geotechnique, 13(3), pp. 177-197 (1963).

50. Gath, I. and Geva, A.B. "Unsupervised optimal fuzzy clustering", Pattern Analysis and Machine Intelligence, IEEE Transactions on, 11(7), pp. 773-780 (1989).

51. Pal, N.R. and Bezdek, J.C. "On cluster validity for the fuzzy c-means model", Fuzzy Systems, IEEE Transactions on, 3(3), pp. 370-379 (1995).

52. Krishnapuram, R. and Freg, C.-P. "Fitting an unknown number of lines and planes to image data through compatible cluster merging", Pattern Recognition, 25(4), pp. 385-400 (1992).

53. Kaymak, U. and Babuska, R. "Compatible cluster merging for fuzzy modelling", Fuzzy Systems, 1995. International Joint Conference of the Fourth IEEE International Conference on Fuzzy Systems and The Second International Fuzzy Engineering Symposium., Proceedings of 1995 IEEE Int, 2(1), pp. 897-904 (1995).

54. Alvarez Grima, M. and Babuška, R. "Fuzzy model for the prediction of unconfined compressive strength of rock samples", International Journal of Rock Mechanics and Mining Sciences, 36(3), pp. 339-349 (1999).

55. Pradhan, B., Sezer, E.A., Gokceoglu, C., and Buchroithner, M.F. "Landslide susceptibility mapping by neuro-fuzzy approach in a landslide-prone area (Cameron Highlands, Malaysia)", Geoscience and Remote Sensing, IEEE Transactions on, 48(12), pp. 4164-4177 (2010).

56. Monjezi, M., Ghafurikalajahi, M., and Bahrami, A. "Prediction of blast-induced ground vibration using artificial neural networks", Tunnelling and Underground Space Technology, 26(1), pp. 46-50 (2011).

57. Smith, G.N., Probability and Statistics in Civil Engineering: An Introduction, Collins London (1986).

58. Jang, J.S.R. and Gulley, N. "Fuzzy logic toolbox user's guide", The Mathworks Inc, Natick (2000).

\section{Biographies}

Hossein Rahnema is an Assistant Professor in Civil and Environmental Engineering at Shiraz University of Technology. He received all of his educational degrees in Shiraz, Iran. He obtained a PhD in Geotechnical Engineering from Civil Engineering Department of Shiraz University, Shiraz, Iran in 2002. His research field was centered on unsaturated soil. Currently, his primary field of research interest is natural hazards engineering. Within this broad field, he has interests in geotechnical earthquake engineering, including soilstructure interaction and surface wave method as well as seismic hazard analysis, damage detection and land subsidence. He is interested in fabricating a new seismic apparatus and performing field seismic tests 
with numerical and analytical modelling to investigate his aforementioned research interests.

Mehdi Hashemi Jokar received a BS degree in Civil Engineering from Tabriz University and MSc degree in Geotechnical Engineering from Graduate University of Advanced Technology, first rank student in both degrees. He is currently a $\mathrm{PhD}$ candidate in Geotechnical Engineering at Shiraz University of Technology, Shiraz, Iran. Because he was the best student among the Iranian top students, he was accepted in Iranian government's scholarship in 2013. He is interested in determination of the characteristics of soil, especially soft clays, experimental work on soil, modelling and evaluation of the soil samples and also design, and construction of cells for soil experiments. During his MSc program, he studied the cells that measure soils' characteristics (besides, he worked on his thesis about designing and constructing a cell for determination of soil characteristics such as swelling parameters with different moisture and pressure levels and also determination of structures foundation reaction built on expansive soils). He has some experience in designing a cell able to measure soils' characteristics, especially in various moisture and pressure levels. Some part of the cell has been completed; however, it has not been completed yet. He has exclusively studied Fuzzy Logic and Adaptive Neuro-Fuzzy Inference System (ANFIS).
He is adept at MATLAB software and has written MATLAB codes that are able to solve engineering problems on optimum condition of ANFIS. It is possible to predict soil characteristics by ANFIS wrote codes with high accuracy.

Hadi Khabbaz is an Associate Professor in Geotechnical Engineering at the University of Technology Sydney (UTS). He received his $\mathrm{PhD}$ degree from University of New South Wales in 1997, Australia. Since graduation, he has taken an active role in conducting practical research on problematic soils and ground improvement techniques. His research has been focused on soft soils, expansive soils, granular particles, and unsaturated porous media. His fundamental and pioneering research on unsaturated soil mechanics has been considered as a significant contribution to the field. His research output and publications on geotechnical aspects of rail track formation are also extensive, as he was a CRC-Rail researcher at University of Wollongong, Australia over five years. He is an assessor and a technical reviewer of the Australian Research Council and the Office for Learning and Teaching grants and many international journals. Since 2013, he has been the Deputy Chair and Chair of the Australian Geomechanics Society (Sydney Chapter). In 2011, he received the Australian Learning and Teaching Council Award for his innovative teaching at UTS. 